\title{
Analytical approach to an integrate-and-fire model with spike-triggered adaptation
}

\author{
Tilo Schwalger ${ }^{1,2, *}$ and Benjamin Lindner, ${ }^{2,3}$ \\ ${ }^{1}$ Brain Mind Institute, École Polytechnique Féderale de Lausanne (EPFL) Station 15, CH-1015 Lausanne, Switzerland \\ ${ }^{2}$ Bernstein Center for Computational Neuroscience, Haus 2, Philippstraße 13, 10115 Berlin, Germany \\ ${ }^{3}$ Department of Physics, Humboldt Universität zu Berlin, Newtonstraße 15, 12489 Berlin, Germany
}

(Received 31 May 2015; published 7 December 2015)

\begin{abstract}
The calculation of the steady-state probability density for multidimensional stochastic systems that do not obey detailed balance is a difficult problem. Here we present the analytical derivation of the stationary joint and various marginal probability densities for a stochastic neuron model with adaptation current. Our approach assumes weak noise but is valid for arbitrary adaptation strength and time scale. The theory predicts several effects of adaptation on the statistics of the membrane potential of a tonically firing neuron: (i) a membrane potential distribution with a convex shape, (ii) a strongly increased probability of hyperpolarized membrane potentials induced by strong and fast adaptation, and (iii) a maximized variability associated with the adaptation current at a finite adaptation time scale.
\end{abstract}

DOI: 10.1103/PhysRevE.92.062703

PACS number(s): 87.19.1c, 05.40.-a, 87.18.Tt

\section{INTRODUCTION}

Nonequilibrium systems with significant fluctuations are encountered in diverse scientific areas such as laser physics and neurobiology. Stochastic differential equations (Langevin equations) and their associated Fokker-Planck equation for the probability density provide a well-established framework for the description of such systems. However, for systems far away from thermodynamic equilibrium, analytical solutions for even the simplest statistics such as the steady-state probability density are known in only a few cases.

A well-known class of nonequilibrium models that are difficult to treat analytically are limit-cycle systems, i.e., multidimensional systems that display an asymptotic periodic solution. In the presence of noise, the condition of detailed balance is violated [1] and the steady-state probability density can be calculated only for particularly simple (symmetric) cases [2] or approximated in the case of a pronounced timescale separation between variables $[3,4]$. Note that for such models one is interested not only in the probability density but also in the mean rotation frequency (or rate) and the irregularity of the round-trip time.

An excellent example of a noisy system out of equilibrium is the nerve cell (neuron), which are the basic units of information transmission and processing in the brain. If subjected to a constant input current or other stereotypical stimuli, they often display periodic firing, i.e., they generate the small stereotypical depolarizations of the membrane potential which are known as action potentials or spikes. In this tonic firing regime of repeated spiking, the action potential can be regarded as a projection of a limit-cycle motion in a space that is spanned by the voltage and one or more gating variables of voltage-dependent ion channels [5]. This motion is noisy because of different sources of fluctuations such as channel noise, synaptic noise, and quasirandom input from other nerve cells, and consequently, the generated spike train can be described as a stochastic point process [6].

\footnotetext{
*tilo.schwalger@epfl.ch
}

While stochastic spiking can be biophysically well described in the frame of a stochastic Hodgkin-Huxley model, not much analytical progress can be made with this kind of model and even fitting it to limited experimental data may be difficult. Integrate-and-fire (IF) models [7,8] are one-dimensional caricatures of neural limit-cycle behavior or of neural excitability, in which a simple reset mechanism is employed: upon reaching a threshold, the generation of a spike is postulated and the voltage variable is reset to a lower value. Despite its simplicity, this class of models and extensions of it can capture the firing times and subthreshold membrane voltage of some neurons under noisy current stimulation surprisingly well $[9,10]$. Furthermore, the voltage probability density, spike-train statistics, and interspike interval (ISI) statistics can be either analytically calculated or efficiently numerically computed.

Ironically, with the aim of better understanding the firing statistics of real cells, researchers have more recently extended IF neurons to multidimensional models, keeping only the fire-and-reset mechanism. Additional variables may describe synaptic filtering [11], external sources of colored noise [12,13], dendritic compartments [14], and subthreshold oscillations [15].

Particularly important in order to mimic the firing of real cells seems to be the inclusion of slow ionic currents that mediate spike-frequency adaptation, a ubiquitous phenomenon across the nervous system [16]. In conjunction with fast fluctuations, adaptation currents shape spontaneous spiking by generating ISI correlations [17-20]. In response to time-dependent stimuli, spike-frequency adaptation endows neurons with computational flexibility and may enable them to respond rapidly to novel features of the stimulus $[16,18,19]$.

Analytical approaches to stochastic IF neurons with spikefrequency adaptation are often limited to the case of slow adaptation [17,21-26]. Our own recent contributions on the perfect IF (PIF) [27] and a general multidimensional IF model [20] with adaptation focus on the calculation of the serial correlation coefficient in the weak-noise limit. Other statistics, such as the probability density, have not been addressed so far (except for the adiabatic treatment in [26]), despite the accessibility of membrane potential distributions 
in experiments [26,28-34] and the importance of this model class.

Here we present a detailed weak-noise approximation for the PIF model with spike-triggered adaptation. The PIF model provides a valid description of a tonically firing neuron for a sufficiently high base current. It has proven successful in matching the spontaneous spiking statistics of sensory neurons $[35,36]$, but also that of more complicated stochastic neuron models [35,37]. Our aim here is to extend the approach presented in [27] in order to compute the probability density of membrane voltage and adaptation current in the case of weak noise but for an arbitrary time scale of the adaptation variable. Our study can also be seen as an important example of a nonpotential system lacking detailed balance, in which the probability density in the weak-noise limit can be calculated analytically without invoking a separation of time scales.

Our paper is organized as follows. In the next section, we introduce in detail the model and statistics of interest. We then consider the deterministic dynamics of the adapting PIF model in Sec. III. In Sec. IV we formulate the stochastic problem in terms of a Fokker-Planck equation and its nontrivial boundary conditions. Here we also sketch the derivation of our solution, which is largely based on the Wentzel-Kramers-Brillouin (WKB) theory but also involves the matching of boundary conditions which are due to the nature of the reset mechanisms for the voltage and adaptation variable. We compare our results to those of numerical simulations of the model in Sec. V for the joint density along the deterministic limit cycle and for the marginal probability densities for the membrane voltage and for the adaptation variable upon firing. We conclude with a brief summary in Sec. VI and present further details of our analytical calculation in the Appendix.

\section{MODEL AND QUANTITIES OF INTEREST}

We consider the white-noise-driven PIF model with a spike-triggered adaptation current [27,38,39]. In this model, the dynamics of the membrane potential $V$ and the adaptation current $a$ are given by

$$
\begin{aligned}
\dot{V} & =\mu-a+\sqrt{2 D} \xi(t), \\
\tau_{a} \dot{a} & =-a+\tilde{\Delta} y(t),
\end{aligned}
$$

where the Gaussian white noise $\xi(t)$ with correlation function $\left\langle\xi(t) \xi\left(t^{\prime}\right)\right\rangle=\delta\left(t-t^{\prime}\right)$ represents fast channel noise, a massive synaptic input from surrounding neurons, or simply a timedependent current injected experimentally. The right-hand side of Eq. (1a) does not depend explicitly on the voltage (we deal here with a PIF neuron), but only via a fire-and-reset rule: whenever $V(t)$ reaches the threshold value $V_{\text {th }}>0$, a spike is emitted and the membrane potential is reset to $V=0$. This defines the spike times $\left\{t_{i}\right\}$ and the output spike train

$$
y(t)=\sum_{i} \delta\left(t-t_{i}\right)
$$

The spike train affects the adaptation variable via Eq. (1b): an output spike leads to a jump by $\Delta=\tilde{\Delta} / \tau_{a}$ in $a$, while between spikes the adaptation variable decays exponentially with the time constant $\tau_{a}$. For an illustration of the model [deterministic paths of both $V(t)$ and $a(t)$ ], see Fig. 1(a). (a)
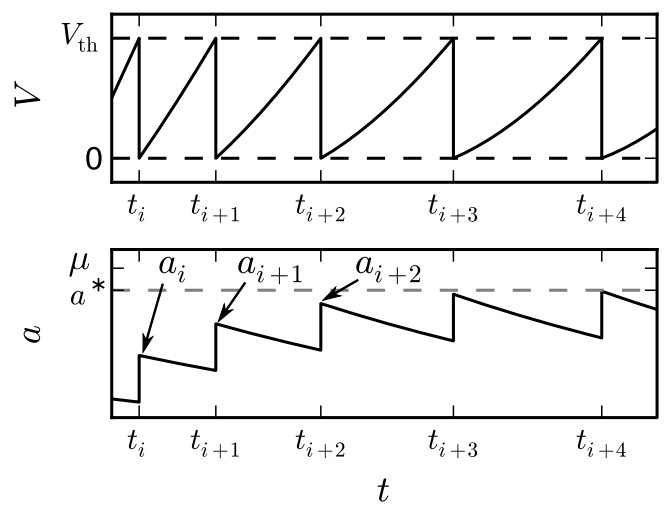

(b)

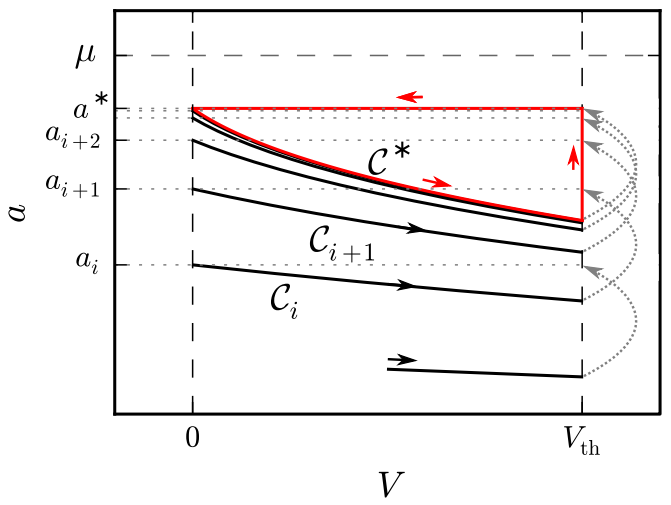

FIG. 1. (Color online) Deterministic dynamics and limit cycle. (a) Typical trajectories $V(t)$ (upper panel) and $a(t)$ (lower panel) for $D=0$. Values of $a(t)$ immediately after the jumps define the sequence $\left\{a_{i}\right\}$ as indicated. This sequence converges to the fixed point $a^{*}$ (dashed line). Note that in this example $a^{*}<\mu$. (b) Corresponding trajectory in the phase plane: the system moves along the curves $\mathcal{C}_{i}$ that start at $a_{i}$ on the reset line and end at the threshold line (dashed vertical lines). At this point a jump occurs to the initial values of the successive curve $\mathcal{C}_{i+1}$. For better visibility, we plot vertical and horizontal lines corresponding to the reset events only for the asymptotic curve $\mathcal{C}^{*}$, i.e., the limit cycle. Parameter values: $\tau_{a}=3, \tilde{\Delta}=3, D=0$.

The main quantity of interest in this paper is the probability density,

$$
P(V, a, t)=\langle\delta(V-V(t)) \delta(a-a(t))\rangle,
$$

in which $a(t)$ and $V(t)$ (with a time argument) indicate the stochastic variables governed by the Langevin equations, Eq. (1), whereas $a$ and $V$ (without a time argument) are the independent variables of the probability density. We also discuss the marginal density of the membrane voltage,

$$
P(V, t)=\int_{0}^{\infty} d a P(V, a, t),
$$

a quantity that can be compared to experimental data. Note that the adaptation variable is usually not accessible.

Of course, spike train and interval statistics are likewise important and may be even more easily comparable with experiments in which only the spike times but not the membrane voltage can be determined. The most important statistics is the firing rate, directly defined by an ensemble 
average of the spike train,

$$
r(t)=\langle y(t)\rangle,
$$

but also related to the probability density—or, more specifically, the probability current—as outlined below.

\section{PHASE-PLANE ANALYSIS OF THE DETERMINISTIC SYSTEM}

We assume that at time $t=t_{i}$ the neuron has fired a spike and has been reset instantaneously, i.e., $V\left(t_{i}\right)=0$ and $a\left(t_{i}\right)=$ $a_{i}$ [cf. Fig. 1(a)]. Without noise the integration of the system, Eq. (1), yields

$$
\begin{gathered}
a(t)=a_{i} \exp \left(-\frac{t-t_{i}}{\tau_{a}}\right), \\
V(t)=\mu \cdot\left(t-t_{i}\right)-\tau_{a} a_{i}\left[1-\exp \left(-\frac{t-t_{i}}{\tau_{a}}\right)\right] .
\end{gathered}
$$

This solution holds true for the interval $t \in\left[t_{i}, t_{i}+T_{\operatorname{det}}\left(a_{i}\right)\right]$, where $T_{\operatorname{det}}\left(a_{i}\right)$ is the deterministic ISI needed to reach the threshold, i.e., $V(t)=V_{\text {th }}$ at time $t=t_{i}+T_{\text {det }}\left(a_{i}\right)$. From Eq. (7) it can be inferred that $T_{\text {det }}\left(a_{i}\right)$ is the solution of the transcendental equation

$$
V_{\mathrm{th}}=\mu T_{\mathrm{det}}\left(a_{i}\right)-\tau_{a} a_{i}\left[1-\exp \left(-\frac{T_{\mathrm{det}}\left(a_{i}\right)}{\tau_{a}}\right)\right] .
$$

At the next spike time $t_{i+1} \equiv t_{i}+T_{\mathrm{det}}\left(a_{i}\right)$ the adaptation variable $a(t)$ is incremented by an amount $\Delta$ and $V$ is reset to 0 . This results in the new initial values $a\left(t_{i+1}\right)=a_{i+1}$ and $V\left(t_{i+1}\right)=0$. Subsequent initial values are related according to

$$
a_{i+1}=a_{i} \exp \left(-\frac{T_{\mathrm{det}}\left(a_{i}\right)}{\tau_{a}}\right)+\Delta .
$$

From this, the trajectory $[a(t), V(t)]$ in the next interval, $t \in\left[t_{i+1}, t_{i+1}+T_{\operatorname{det}}\left(a_{i+1}\right)\right]$, is obtained by replacing $i$ with $i+1$ in Eq. (6) and Eq. (7). A trajectory obtained by repeating this procedure is illustrated in Fig. 1(b). Reset and threshold potentials correspond to vertical lines in the phase plane. We refer to the piece of the trajectory within a specific time interval, $t \in\left[t_{i+1}, t_{i+1}+T_{\operatorname{det}}\left(a_{i+1}\right)\right]$, as $\mathcal{C}_{i}$. Each curve $\mathcal{C}_{i}$ is completely determined by its initial value $a_{i}$. Hence, it is sufficient to study the sequence $\left\{a_{i}\right\}$ instead of the full dynamics $a(t)$ and $V(t)$.

As $i \rightarrow \infty$, the sequence of curves $\mathcal{C}_{i}$ converges to a limit curve $\mathcal{C}^{*}$ [cf. Fig. 1(b)], which can be regarded as the essential part of a limit cycle. The convergence of trajectories to $\mathcal{C}^{*}$ has a counterpart in the convergence of the sequence $\left\{a_{i}\right\}$ to a limiting value $a^{*}$. Thus, $\mathcal{C}^{*}$ is attracting if the map, (9), possesses a stable fixed point $a^{*}$, which requires that $a_{i+1}=$ $a_{i}=a^{*}$ or

$$
a^{*}=a^{*} \exp \left(-\frac{T^{*}}{\tau_{a}}\right)+\Delta .
$$

Here, $T^{*} \equiv T_{\text {det }}\left(a^{*}\right)$ denotes the ISI if the system is on the limit cycle $\mathcal{C}^{*}$. This interval has to fulfill Eq. (8) evaluated at $a_{i}=a^{*}$ :

$$
V_{\mathrm{th}}=\mu T^{*}-\tau_{a} a^{*}\left[1-\exp \left(-\frac{T^{*}}{\tau_{a}}\right)\right] .
$$

(a)

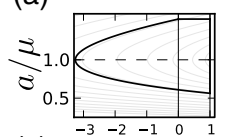

(c)

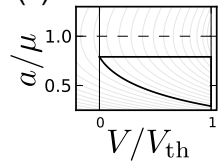

(b)

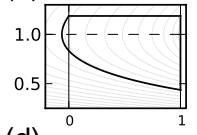

(d)

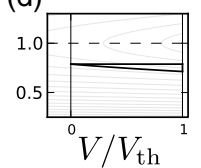

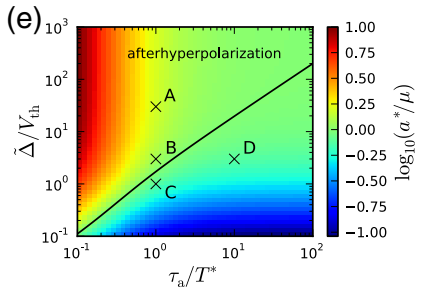

FIG. 2. (Color online) Dependence of the limit cycle on the system parameters. Examples of limit cycles are shown in the phase plane (a-d) by solid lines for parameter sets A-D as indicated in (e). Reset and threshold lines are represented by thin solid lines; the dashed line represents the nullcline $a=\mu$, where $\dot{V}=0$. (e) The fixed point $a^{*}$ normalized by $\mu=\left(V_{\mathrm{th}}+\tilde{\Delta}\right) / T^{*}$ in the parameter space spanned by the adaptation time constant $\tau_{a}$ and the adaptation strength $\tilde{\Delta}$. The solid line divides the parameter space into two regions, corresponding to $a^{*}>\mu$ (afterhyperpolarization regime; upper region) and $a^{*}<\mu$ (lower region).

Solving Eq. (10) and Eq. (11) yields

$$
T^{*}=\frac{V_{\text {th }}+\tilde{\Delta}}{\mu}, \quad a^{*}=\frac{\Delta}{1-\exp \left(-T^{*} / \tau_{a}\right)} .
$$

which permits the parametrization of $\mathcal{C}^{*}$

$$
a(t)=a^{*} e^{-t / \tau_{a}}, \quad V(t)=\mu t-\tau_{a} a^{*}\left(1-e^{-t / \tau_{a}}\right)
$$

for $0 \leqslant t \leqslant T^{*}$.

The initial value of $a$ on the limit cycle, $a^{*}$, is important because in a system with noise $a^{*}$ sets the order of magnitude of the peak currents $a_{i}$, which are distributed in the neighborhood of $a^{*}$. Furthermore, $a^{*}$ defines two regimes, depending on whether $a^{*}$ is smaller or larger than the base current $\mu=\left(V_{\mathrm{th}}+\right.$ $\tilde{\Delta}) / T^{*}$. In Fig. 2(e) the magnitude of $a^{*}$ normalized by $\mu$ is displayed as a function of the system parameters $\tau_{a} / T^{*}$ and $\tilde{\Delta} / V_{\mathrm{th}}$ of the deterministic system. The two regimes mentioned before are separated by the line

$$
\frac{\tilde{\Delta}}{V_{\mathrm{th}}}=\frac{1-e^{-\delta}}{\delta-1+e^{-\delta}}, \quad \delta=\frac{T^{*}}{\tau_{a}} .
$$

Above this line (strong adaptation), the limit cycle exhibits an excursion to negative membrane potentials $V(t)$ [afterhyperpolarization; Fig. 2(a) and 2(b)]. Below the separating line, i.e., for $a^{*} / \mu<1$, the drift on the limit cycle is always positive [Figs. 2(c) and 2(d)]. In this case the limit cycle does not go below the reset voltage.

An increase in the adaptation time constant $\tau_{a}$ has two distinct effects [cf. Figs. 2(b) and 2(d)]: First, because $a$ decreases more slowly the phase-space is stretched horizontally. Second, because the jump size $\Delta=\tilde{\Delta} / \tau_{a}$ scales inversely with the adaptation time constant, the jump in $a$ at the reset becomes smaller with increasing $\tau_{a}$.

We, finally, mention that our model can be transformed into a nondimensional form by measuring time in units of the deterministic ISI $T^{*}$ and voltage in units of the threshold $V_{\mathrm{th}}$ : $\bar{t}=t / T^{*}, \bar{V}=V / V_{\text {th }}$, and $\bar{a}=a T^{*} / V_{\text {th }}$. In these units the dynamics reads

$$
\begin{aligned}
& \dot{\bar{V}}=1+\bar{\Delta}-\bar{a}+\sqrt{2 \bar{D}} \xi(\bar{t}), \\
& \bar{\tau}_{\mathrm{a}} \dot{\bar{a}}=-\bar{a}+\bar{\Delta} \sum \delta\left(\bar{t}-\bar{t}_{i}\right),
\end{aligned}
$$


with a rescaled threshold $\bar{V}_{\text {th }}=1$. The system is completely specified by the three dimensionless parameters

$$
\bar{\tau}_{\mathrm{a}}=\frac{\tau_{a}}{T^{*}}, \quad \bar{\Delta}=\frac{\tilde{\Delta}}{V_{\mathrm{th}}}, \quad \bar{D}=D \frac{T^{*}}{V_{\mathrm{th}}^{2}} .
$$

These parameters are reported for the simulations below. From this, the parameters $\mu$ and $\Delta$, which appear in our theoretical expressions, can be inferred as

$$
\mu=(1+\bar{\Delta}) \frac{V_{\mathrm{th}}}{T^{*}}, \quad \Delta=\frac{\bar{\Delta}}{\bar{\tau}_{a}} \cdot \frac{V_{\mathrm{th}}}{T^{*}}
$$

[cf. Eq. (12)]. Without loss of generality, one can choose $V_{\text {th }}=$ $T^{*}=1$, which we have adopted in our simulations, unless stated otherwise.

\section{WKB THEORY FOR THE PROBABILITY DENSITY IN THE LIMIT OF WEAK NOISE}

We now turn to the case of a finite noise intensity and derive an approximate solution of the Fokker-Planck equation corresponding to the Langevin equations, Eq. (1). The essential small parameter of our theory is the noise intensity $D$.

\section{A. Boundary value problem}

The Fokker-Planck equation for the joint probability density $P(V, a, t)$, defined in Eq. (3), in the domain $V<V_{\mathrm{th}}, a>0$, can be written as a continuity equation:

$$
\begin{aligned}
\partial_{t} P(V, a, t)+\partial_{V} J_{V}(V, a, t)+\partial_{a} J_{a}(V, a, t) \\
=\underbrace{-J_{V}\left(V_{\text {th }}-, a, t\right) \delta\left(V-V_{\text {th }}\right)}_{\text {absorption at } V=V_{\text {th }}} \\
+\underbrace{J_{V}\left(V_{\text {th }}-, a-\Delta, t\right) \delta(V)}_{\text {jump and reinsertion at } V=0} .
\end{aligned}
$$

Here, the notation $f(x-)$ and $f(x+)$ indicates the leftand right-side limit, respectively. In Eq. (19) $J_{V}(V, a, t)$ and $J_{a}(V, a, t)$ are the components of the two-dimensional probability current $\mathbf{J}=\left(J_{V}, J_{a}\right)$, defined by

$$
\begin{gathered}
J_{V}(V, a, t)=\left[(\mu-a)-D \partial_{V}\right] P(V, a, t), \\
J_{a}(V, a, t)=-\frac{a}{\tau_{a}} P(V, a, t) .
\end{gathered}
$$

The right side of Eq. (19) represents the source and sink terms: trajectories that reach the threshold at point $\left(V_{\mathrm{th}}, a-\Delta\right)$ are absorbed (sink) and are reinjected at point $(0, a)$ (source).

As an alternative to Eq. (19) we can write the Fokker-Planck equation without sink and source terms,

$$
\partial_{t} P(V, a, t)=\left[-(\mu-a) \partial_{V}+\frac{1}{\tau_{a}} \partial_{a} a+D \partial_{V}^{2}\right] P(V, a, t),
$$

and, additionally, impose boundary conditions at $V=0$ and $V=V_{T}$ as explained in the following.

Because the process is driven by white noise in the voltage variable, $P(V, a, t)$ has to be continuous with respect to $V$ at every point and, in particular, at the reset voltage:

$$
P(0-, a, t)=P(0+, a, t) .
$$

Furthermore, the sink at the threshold implies that there is no probability beyond the threshold, which, combined with the continuity equation, implies that

$$
P\left(V_{\mathrm{th}}, a, t\right)=0 .
$$

Integrating the continuity equation, Eq. (19), about the reset potential from $V=-\epsilon$ to $V=\epsilon$ and letting $\epsilon \rightarrow 0$ yields the condition

$$
J_{V}(0+, a, t)-J_{V}(0-, a, t)=J_{V}\left(V_{\mathrm{th}}, a-\Delta, t\right) .
$$

Using the definition of the current $J_{V}$, Eq. (20), and accounting for the continuity and the absorbing boundary, Eqs. (23) and (24), the latter condition becomes

$$
\left.\partial_{V} P(V, a, t)\right|_{0+}-\left.\partial_{V} P(V, a, t)\right|_{0-}=\left.\partial_{V} P(V, a-\Delta, t)\right|_{V_{\mathrm{th}}} .
$$

This discontinuity in the derivative $\partial_{V} P$ realizes the jump and reset. Furthermore, all probability and currents should vanish at infinity, which leads to the natural boundary conditions

$$
\begin{aligned}
\lim _{V \rightarrow-\infty} P(V, a, t) & =0, \quad \lim _{a \rightarrow \infty} P(V, a, t)=0, \\
\lim _{V \rightarrow-\infty} \partial_{V} P(V, a, t) & =0 .
\end{aligned}
$$

Finally, the probability density must be normalized:

$$
\int_{-\infty}^{V_{\mathrm{th}}} \mathrm{d} V \int_{0}^{\infty} \mathrm{d} a P(V, a, t)=1
$$

We do not specify initial conditions except for the constraint that the density is confined to $\left\{V, a \mid V \leqslant V_{\text {th }}, a>0\right\}$.

In the following we focus on the stationary probability density $P_{0}(V, a)$, which is approached in the limit $t \rightarrow \infty$ and obtained by setting the time derivative in the Fokker-Planck equation to 0 . Knowledge of the stationary solution permits us to determine the following important statistics of the system:

(1) The stationary firing rate,

$$
r_{0}=\int_{0}^{\infty} \mathrm{d} a J_{V}^{(s)}\left(V_{\mathrm{th}}, a\right)=-D \int_{0}^{\infty} \mathrm{d} a \partial_{V} P_{0}\left(V_{\mathrm{th}}, a\right),
$$

where the stationary probability current $J_{V}^{(s)}=[(\mu-a)-$ $\left.D \partial_{V}\right] P_{0}(V, a)$ has been used.

(2) The distribution of the subthreshold membrane potential,

$$
P_{0}(V)=\int_{0}^{\infty} \mathrm{d} a P_{0}(V, a) .
$$

(3) The distribution of the adaptation current upon firing,

$$
P_{\mathrm{f}}(a) \propto J_{V}^{(s)}\left(V_{\mathrm{th}}, a-\Delta\right)=-D \partial_{V} P_{0}\left(V_{\mathrm{th}}, a-\Delta\right) .
$$

Even in the stationary case the two-dimensional FokkerPlanck equation cannot be solved exactly. The essential assumption for our approximate solution presented in the following is a weak noise. It is convenient to introduce a small, nondimensional parameter $\epsilon$ that scales the noise intensity as

$$
D=\epsilon \hat{D}
$$

where $\hat{D}$ is of order 1 and carries the physical dimension of the parameter. Because of this slight change in notation and for the convenience of the reader, we specify the Fokker-Planck 
equation and its boundary conditions for the stationary case. The equation itself reads

$$
-(\mu-a) \partial_{V} P_{0}+\frac{1}{\tau_{a}} \partial_{a}\left(a P_{0}\right)+\epsilon \hat{D} \partial_{V}^{2} P_{0}=0 .
$$

The boundary conditions, Eqs. (23)-(26), lead in the stationary case to the following equations:

$$
\begin{gathered}
P_{0}\left(V_{\mathrm{th}}, a\right)=0, \\
P_{0}(0-, a)=P_{0}(0+, a), \\
\partial_{V} P_{0}(0+, a)-\partial_{V} P_{0}(0-, a)=\partial_{V} P_{0}\left(V_{\mathrm{th}}, a-\Delta\right), \\
\lim _{V \rightarrow-\infty} P_{0}(V, a)=0, \\
\lim _{a \rightarrow \infty} P_{0}(V, a)=0, \\
\lim _{V \rightarrow-\infty} \partial_{V} P_{0}(V, a)=0 .
\end{gathered}
$$

The solution of this set of equations is complemented by the normalization condition, Eq. (27). In our weak-noise approximation it is, however, also possible to determine the free multiplicative factor (usually fixed by normalization) as follows. In the limit $\epsilon \rightarrow 0$, the firing rate is given by $1 / T^{*}$. Assuming that a weak noise does not change the firing rate, we obtain, using Eq. (28), an additional boundary condition,

$$
-\epsilon \hat{D} \int_{0}^{\infty} \mathrm{d} a \partial_{V} P_{0}\left(V_{\mathrm{th}}, a\right)=\frac{1}{T^{*}},
$$

which completes the boundary value problem for $P_{0}(V, a)$.

For weak noise, we solve the Fokker-Planck equation, (32), in different regions. These regions are sketched in Fig. 3; they depend on whether $a^{*}<\mu$ or $a^{*}>\mu$. The solutions in different regions are matched according to the continuity and reset condition. Specifically, for $a^{*}<\mu$ [Fig. 3(a)] the equation is solved separately below the reset line $(V<0$; region I) and between the reset and the threshold line $(0<V \leqslant$ $V_{\text {th }} ;$ region II). The division of the plane is different for $a^{*}>\mu$ as indicated in Fig. 3(b). In both cases, the solution in region II is further subdivided into a solution within a thin boundary layer in the vicinity of the threshold ("inner solution") and beyond ("outer solution"). The outer solution can be obtained within the frame of WKB theory, yielding the asymptotic (a)

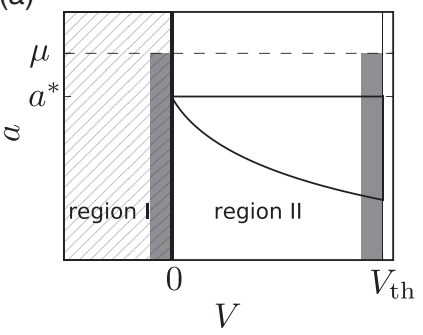

(b)

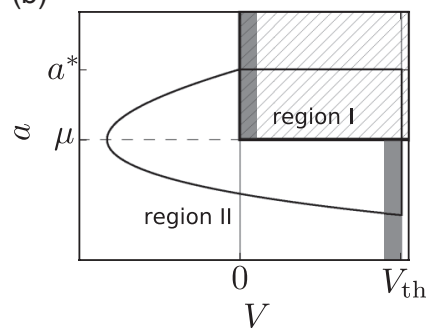

FIG. 3. Different regions of solutions and locations of the boundary layers. (a) Case $a^{*}<\mu$; (b) case $a^{*}>\mu$. The solution is calculated separately for region I (hatched area) and region II (white area). In boundary layers of thickness $\mathcal{O}(\epsilon)$ [indicated by shaded (gray) areas], the solution drops off rapidly. form of the probability density around the deterministic limit cycle $\mathcal{C}^{*}$. In the deterministic limit, the probability density is proportional to a $\delta$ function along the limit cycle. The WKB solution describes the noise-induced softening of this $\delta$ singularity by approximating the probability density by a Gaussian perpendicular to the limit cycle-a Gaussian with a variance that is proportional to $\epsilon$.

\section{B. Stationary solution of the Fokker-Planck equation}

In the following we sketch the derivation of the stationary solution; details are presented in the Appendix (Sec. A). In region I, the Fokker-Planck equation is solved in perturbation theory. Using the stretched variable $x=V / \epsilon$ we avoid the singular behavior of $P_{0}(V, a)$ at $V=0$ (reset) in the limit $\epsilon \rightarrow 0$ (Fig. 4). The leading-order solution in region I can be
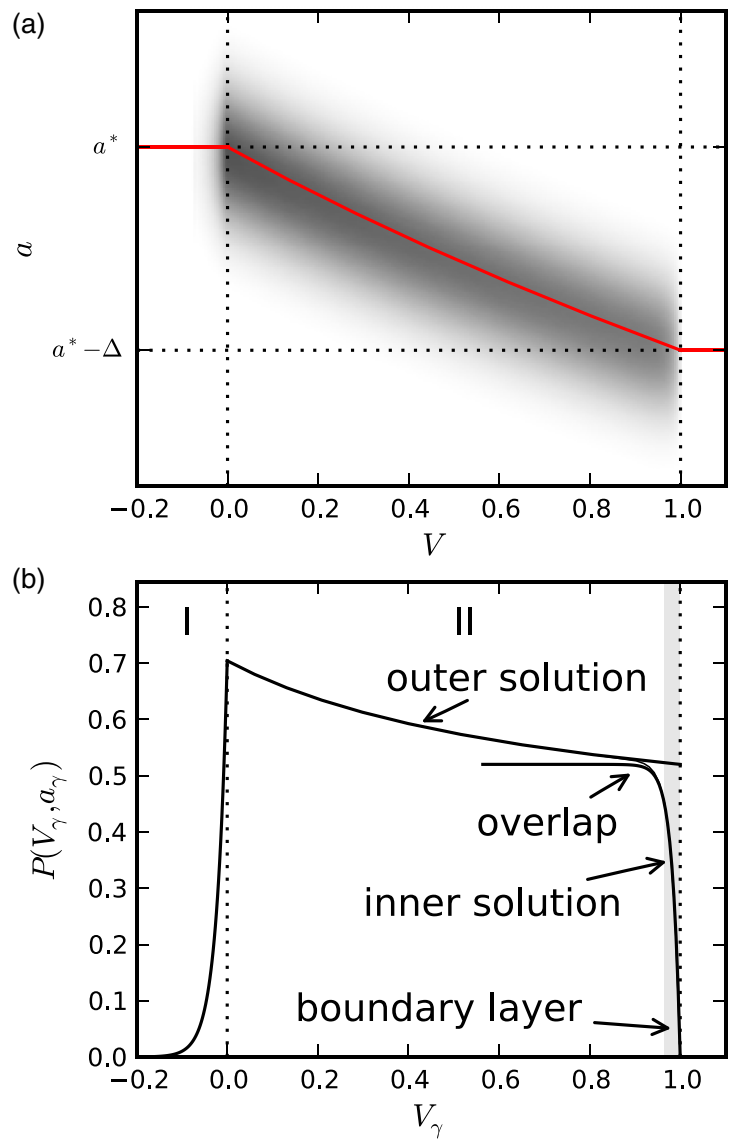

FIG. 4. (Color online) Structure of the stationary solution of the Fokker-Planck equation. (a) Stationary probability density in the vicinity of the limit cycle. (b) The density along the path of the solid (red) line $\gamma$ is shown. The plot illustrates the different regions of solutions and indicates how the solutions are patched together. In region I (corresponding to $V<0$ in the case $a^{*}<\mu$ ) the solution rises steeply in a boundary layer of thickness $\mathcal{O}(\epsilon)$ below the reset. In region II, We use a WKB ansatz for the solution, which must be continuous at $V=0$. This ansatz cannot, however, capture the steep decline to 0 in a second boundary layer close to the threshold (absorbing boundary). The inner solution of this boundary layer is asymptotically matched to the WKB solution (outer solution). 
written as (Sec. A 1)

$$
P_{0, \mathrm{I}}(V, a)=\frac{M(a)}{\sqrt{\epsilon} \lambda(a)} \exp \left(\frac{\lambda(a) V}{\epsilon}\right)
$$

with

$$
\lambda(a)=\frac{\mu-a}{\hat{D}},
$$

and $M(a)$ is a function that is determined by the continuity condition, Eq. (34).

In region II, boundary layer theory [40] is used to find separate solutions outside and inside the boundary layer, which are then matched asymptotically. The inner solution, i.e., the solution close to the threshold $\left(0 \ll V \leqslant V_{\text {th }}\right)$, is obtained as in region I: Using the stretched variable $x=\left(V-V_{\text {th }}\right) / \epsilon$ the Fokker-Planck equation is solved perturbatively, which yields (Sec. A 2)

$$
P_{0, \mathrm{II}, \mathrm{in}}(V, a)=\frac{N(a)}{\sqrt{\epsilon} \lambda(a)}\left[\exp \left(\frac{\lambda(a)\left(V-V_{\mathrm{th}}\right)}{\epsilon}\right)-1\right] .
$$

Here, $N(a)$ is determined by the reset condition, Eq. (35).

The outer solution is obtained in the WKB approximation (see, e.g., [41]).We make the following ansatz for the outer solution:

$$
P_{0, \mathrm{II}, \text { out }}(V, a)=\frac{1}{\sqrt{2 \pi \epsilon}} \Psi(V, a) \exp \left(-\frac{\Phi(V, a)}{\epsilon}\right) .
$$

The functions $\Phi(V, a)$ and $\Psi(V, a)$ are independent of $\epsilon$ and can be regarded as a nonequilibrium potential [42] and a prefactor, respectively. Both functions obey first-order, nonlinear partial differential equations, to be solved by the method of characteristics. Further constraints on $\Phi(V, a)$ ensure that the nonequilibrium potential attains a local minimum on the limit curve $\mathcal{C}^{*}$.

For the calculation it is advantageous to switch to a coordinate system given by the characteristics of the system (see Fig. 5 for an illustration). The new coordinates are denoted $s$ and $\tau$. The variable $s$ distinguishes the characteristic curves $\gamma(s)=(V(\tau ; s), a(\tau ; s))$, whereas $\tau$ can be regarded as a time-like variable describing the propagation along the characteristic curves. Specifically, the initial conditions read $a(\tau=0 ; s)=s$ and $V(\tau=0 ; s)=0$ (see Fig. 5 and Appendix, Sec. A 2, for more details). For $s=a^{*}$, the characteristic curve coincides with the deterministic limit curve $\mathcal{C}^{*}$ and $\tau$ plays the role of time $t$ in the limit-cycle parametrization, Eq. (13). However, for $s \neq a^{*}$, the characteristic curve does not coincide, in general, with a trajectory starting at $V=0$ and $a=s$. Using these coordinates, the method of characteristics yields the parametric solution (Appendix, Sec. A 2)

$$
\begin{gathered}
V(\tau, s)=\left[\mu+2 \hat{D} p_{0}(s)\right] \tau-\tau_{a} s\left(1-e^{-\tau / \tau_{a}}\right), \\
a(\tau, s)=s e^{-\tau / \tau_{a}}, \\
\Phi(V, a)=\hat{D} p_{0}^{2}(s) \tau+\phi_{0}(s), \\
\Psi(V, a)=\psi_{0}(s) e^{\tau / \tau_{a}} \\
\times \sqrt{\frac{\mu-s+2 \hat{D} p_{0}(s)}{2 \hat{D} p_{0}{ }^{\prime}(s) s \tau / \tau_{a}+\mu-s+2 \hat{D} p_{0}(s)}},
\end{gathered}
$$

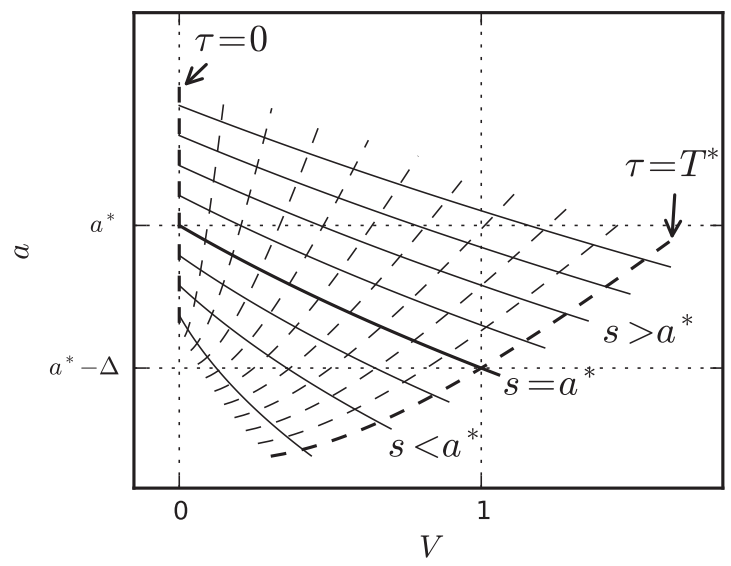

FIG. 5. Parametrization of region II by the characteristic coordinates $\tau$ and $s$. For each value of $s$ there is a characteristic curve parametrized by the coordinate $\tau$ (solid lines). Along these curves the functions $\Phi(V, a)$ and $\Psi(V, a)$ obey ordinary differential equations with respect to the "time" $\tau$. The parameter $s$ corresponds to the initial condition at time $\tau=0$, where, in the original coordinates, $V=0$ and $a=s$. A particularly important characteristic is given by the initial value $s=a^{*}$ : this curve is identical to the limit curve $\mathcal{C}^{*}$, where all probability is concentrated in the deterministic limit. Because the deterministic period is $T^{*}$, the characteristic curve for $s=a^{*}$ crosses the threshold $V=V_{\text {th }}$ at time $\tau=T^{*}$. Dashed lines are isochrons, where $\tau=$ const.

where

$$
p_{0}(s)=-\frac{\mu-s}{2 \hat{D}}+\operatorname{sgn}\left(\mu-a^{*}\right) \sqrt{\frac{(\mu-s)^{2}}{4 \hat{D}^{2}}+\frac{s \phi_{0}^{\prime}(s)}{\hat{D} \tau_{a}}} .
$$

The functions $\phi_{0}(s)=\Phi(0, s)$ and $\psi_{0}(s)=\Psi(0, s)$ are determined by the reset condition and normalization condition, respectively. However, we could not find a solution that obeys these conditions for all $s>0$. Instead, we require that these conditions are satisfied only close to the limit cycle $\left(s=a^{*}\right)$, which is justified for a sufficiently small noise intensity. To this end, we consider the leading order of the functions in $s-a^{*}$ :

$$
\begin{gathered}
\phi_{0}(s)=\frac{\phi_{0}^{\prime \prime}\left(a^{*}\right)}{2}\left(s-a^{*}\right)^{2}+\mathcal{O}\left(\left(s-a^{*}\right)^{3}\right), \\
\psi_{0}(s)=\psi_{0}\left(a^{*}\right)+\mathcal{O}\left(s-a^{*}\right) .
\end{gathered}
$$

Hence, we only have to determine $\phi_{0}^{\prime \prime}\left(a^{*}\right)$ and $\psi_{0}\left(a^{*}\right)$ from the reset and normalization condition, respectively. Because the reset condition also involves the the probability density at the threshold, inner and outer solutions have to be matched asymptotically in a common overlap region (Fig. 4). The final result of these considerations (Appendix, Sec. A 3) is

$$
\phi_{0}^{\prime \prime}\left(a^{*}\right)=\frac{\tau_{a}^{2} \mu \Delta\left[2 a^{*}\left(\mu-a^{*}+\Delta\right)-\mu \Delta\right]}{2 \hat{D} a^{* 2} T^{*}\left(a^{*}-\Delta\right)^{2}} .
$$

Further, by the normalization condition, we obtain (Appendix, Sec. A 4)

$$
\psi_{0}\left(a^{*}\right)=\frac{\sqrt{\phi_{0}^{\prime \prime}\left(a^{*}\right)}}{\left|\mu-a^{*}\right| T^{*}}
$$


and the functions

$$
\begin{aligned}
& M(a)=\frac{\mu-a}{\sqrt{2 \pi} \hat{D}} \psi_{0}\left(a^{*}\right) \exp \left[-\frac{\phi_{0}^{\prime \prime}\left(a^{*}\right)}{2 \epsilon}\left(a-a^{*}\right)^{2}\right], \\
& N(a)=-\frac{\lambda(a)}{\sqrt{2 \pi}} \frac{\left|\mu-a^{*}\right|}{\mu-a^{*}+\Delta} \psi_{0}\left(a^{*}\right) \\
& \times \exp \left(-\frac{\phi_{0}^{\prime \prime}\left(a^{*}\right)}{2 \epsilon}\left(a-a^{*}+\Delta\right)^{2}\right) .
\end{aligned}
$$

Let us summarize the full solution. With Eqs. (40) and (52), the solution in region I reads

$$
P_{0, \mathrm{I}}(V, a)=\frac{\psi_{0}\left(a^{*}\right)}{\sqrt{2 \pi \epsilon}} \exp \left(\frac{\mu-a^{*}}{\epsilon \hat{D}} V-\frac{\phi_{0}^{\prime \prime}\left(a^{*}\right)}{2 \epsilon}\left(a-a^{*}\right)^{2}\right)
$$

In region II, using Eqs. (42) and (53) the inner solution can be written as

$$
\begin{aligned}
P_{0, \mathrm{II}, \text { in }}(V, a)= & \frac{\psi_{0}\left(a^{*}\right)}{\sqrt{2 \pi \epsilon}} \frac{\left|\mu-a^{*}\right|}{\mu-a^{*}+\Delta} \\
& \times\left[1-\exp \left(\frac{\mu-a^{*}+\Delta}{\epsilon \hat{D}}\left(V-V_{\mathrm{th}}\right)\right)\right] \\
& \times \exp \left[-\frac{\phi_{0}^{\prime \prime}\left(a^{*}\right)}{2 \epsilon}\left(a-a^{*}+\Delta\right)^{2}\right] .
\end{aligned}
$$

The outer solution in the neighborhood of the limit cycle reads, in parametric representation,

$$
\begin{aligned}
& P_{0, \mathrm{II}, \text { out }}(V, a) \\
& =\frac{\psi_{0}\left(a^{*}\right) e^{\tau / \tau_{a}}}{\sqrt{2 \pi \epsilon}} \sqrt{\frac{\mu-s+2 \hat{D} p_{0}(s)}{2 \hat{D} p_{0}^{\prime}(s) s \tau / \tau_{a}+\mu-s+2 \hat{D} p_{0}(s)}} \\
& \times \exp \left[-\frac{1}{\epsilon}\left(\hat{D} p_{0}^{2}(s) \tau+\frac{\phi_{0}^{\prime \prime}\left(a^{*}\right)}{2}\left(s-a^{*}\right)^{2}\right)\right], \\
& V(\tau, s)=\left[\mu+2 \hat{D} p_{0}(s)\right] \tau-\tau_{a} s\left(1-e^{-\tau / \tau_{a}}\right), \\
& a(\tau, s)=s e^{-\tau / \tau_{a}}
\end{aligned}
$$

for $0 \leqslant \tau \leqslant T^{*}$ and $p_{0}(s)$ given by

$$
\begin{aligned}
p_{0}(s)= & -\frac{\mu-s}{2 \hat{D}}+\operatorname{sgn}\left(\mu-a^{*}\right) \\
& \times \sqrt{\frac{(\mu-s)^{2}}{4 \hat{D}^{2}}+\frac{s\left(s-a^{*}\right)}{\hat{D} \tau_{a}} \phi_{0}^{\prime \prime}\left(a^{*}\right) .}
\end{aligned}
$$

In the solution variations of $s$ should be considered only in a small interval around $s=a^{*}$, i.e., $s \in\left[a^{*}-\mathcal{O}\left(\epsilon^{1 / 2}\right), a^{*}+\right.$ $\left.\mathcal{O}\left(\epsilon^{1 / 2}\right)\right]$, because outside this neighborhood the probability density is exponentially small. In particular, $s$ should not attain the critical point $s=\mu$ but must be either below or above this point. We would like to emphasize that our weak-noise theory does not cover the singular case $a^{*}=\mu$.

In this section we have obtained an approximation for the full two-dimensional joint probability density in the stationary case. Simplified expressions for various marginal statistics are derived in the following section.

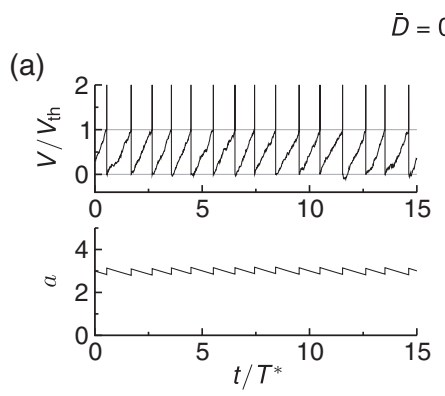

(b)
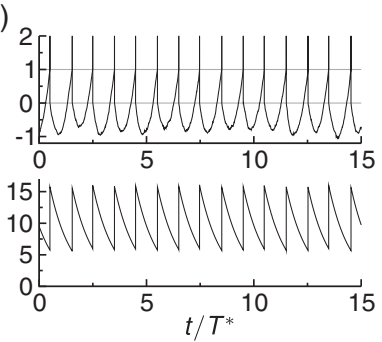

$\bar{D}=0.1$
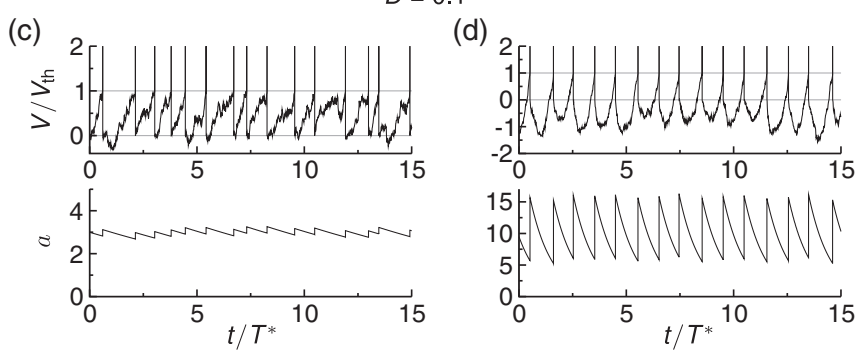

FIG. 6. Sample trajectories in the presence of noise. Trajectories of $V(t)$ (upper panels) and $a(t)$ (lower panels) for a small noise intensity, $\bar{D}=0.01(\mathrm{a}, \mathrm{b})$, and a moderate noise intensity, $\bar{D}=0.1$ (c,d). (a,c) Trajectories for slow and weak adaptation $\left(\bar{\tau}_{a}=10, \bar{\Delta}=\right.$ $3)$; (b,d) trajectories for fast and strong adaptation $\left(\bar{\tau}_{a}=1, \bar{\Delta}=10\right)$. Threshold and reset potentials are indicated by horizontal gray lines. Note that, above threshold, spikes have been explicitly added for better visibility; they do not contribute to the membrane potential distribution.

\section{COMPARISON OF THEORY TO NUMERICAL SIMULATIONS}

We have worked out approximate expressions for the probability density of the two-dimensional stochastic nonpotential system in the limit of weak noise. In order to estimate how "weak" the noise has to be for these approximations to be meaningful, in the following we compare our results to those of extensive numerical simulations, which were obtained as follows. We simulated long trajectories $[V(t), a(t)]$ that contained $N=10^{4}$ and $N=10^{5}$ spikes, from which we built histograms approximating $P_{0}(V)$ and $P_{0}(V, a)$, respectively. Voltages and times are measured in units of $V_{\text {th }}$ and $T^{*}$, respectively. That is, all parameters are nondimensionalized such that $V_{\text {th }}=1$ and $T^{*}=1$. Equation (1) was solved by the Euler-Maruyama method with a time step $\Delta t=10^{-5}$. For a given adaptation strength $\tilde{\Delta}$, we set the base current to $\mu=1+\tilde{\Delta}$ to get $T^{*}=1$ [cf. Eq. (18)]. Short sections of typical trajectories are shown in Fig. 6. For better visibility, the spikes above threshold are added here to highlight the spike train, although they do not contribute to the subthreshold voltage distribution $P_{0}(V)$.

We test our theoretical predictions in three ways. First, we compare the probability density along the deterministic limit cycle, for which we obtain particularly simple expressions. Second, we derive the marginal density with respect to the membrane potential and compare this experimentally accessible statistics with simulation results. Third, we derive the probability density of the adaptation variable upon firing 
and test its agreement with numerical simulations. The latter density is an important statistic because it quantifies the degree of adaptation-induced variability of the ISI.

\section{A. Stationary solution evaluated on the limit cycle}

We first consider the solution on the limit cycle, where the expressions can be considerably simplified. In region I, the solution at $a=a^{*}$ reads

$$
P_{0, \mathrm{I}}\left(V, a^{*}\right)=\frac{\psi_{0}\left(a^{*}\right)}{\sqrt{2 \pi \epsilon}} \exp \left(\frac{\mu-a^{*}}{\epsilon \hat{D}} V\right) .
$$

In region II, the inner solution at $a=a^{*}-\Delta$ is given by

$$
\begin{aligned}
P_{0, \mathrm{II}, \mathrm{in}}\left(V, a^{*}\right)= & \frac{\psi_{0}\left(a^{*}\right)}{\sqrt{2 \pi \epsilon}} \frac{\left|\mu-a^{*}\right|}{\mu-a^{*}+\Delta} \\
& \times\left[1-\exp \left(\frac{\mu-a^{*}+\Delta}{\epsilon \hat{D}}\left(V-V_{\mathrm{th}}\right)\right)\right] .
\end{aligned}
$$

The outer solution on the limit cycle can be expressed as [see Appendix, Sec. A 3, Eqs. (A68) and (A69)]

$$
\begin{aligned}
\left.P_{0, \text { II out }}(V(\tau), a(\tau))\right|_{\mathcal{C}^{*}} & =\frac{\psi_{0}\left(a^{*}\right) e^{\tau / \tau_{a}}}{\sqrt{2 \pi \epsilon(\nu \tau+1)}}, \\
a(\tau) & =a^{*} e^{-\tau / \tau_{a}}, \\
V(\tau) & =\mu \tau-\tau_{a} a^{*}\left(1-e^{-\tau / \tau_{a}}\right),
\end{aligned}
$$

where $\tau \in\left[0, T^{*}\right]$ and

$$
v=\frac{2 \hat{D} a^{* 2} \phi_{0}^{\prime \prime}\left(a^{*}\right)}{\tau_{a}^{2}\left(\mu-a^{*}\right)^{2}} .
$$

We show the stationary probability density along the limit cycle in Figs. 7(b), 7(d), and 7(f); for comparison, the color plots at the left [Figs. 7(a), 7(c), and 7(e)] also display the joint density in the phase plane together with the deterministic limit cycle. As expected for weak noise [Fig. 7(b)], our analytical results agrees well with the simulation result [Fig. 7(b)]. For a larger noise intensity [Fig. 7(d)], we observe quantitative deviations, although our theory still captures qualitatively the change in the density, e.g., the broadening of the distribution in region I and the smoother shape of the inner solution in region II. The quantitative deviation of the outer solution in region II from our prediction is most likely due to the fact that the maximum of the probability density function is not attained on the deterministic limit cycle [cf. Fig. 7(c)], contrary to what we assumed in our WKB approximation. A shift of the maximum away from the limit cycle could appear in our theory if higher-order terms of $\Psi$ were taken into account.

While Figs. 7(a)-7(d) explore the simple case $a^{*}<\mu$, Fig. 7(f) demonstrates that our theory also works for $a^{*}>\mu$, i.e., for strong adaptation. In this case, the density along $\mathcal{C}^{*}$ is not a unique function of $V$ anymore and we have to consider two functions corresponding to different segments of the deterministic limit cycle. Both functions show a quantitative agreement between theory and simulation results.

\section{B. Distribution of the membrane potential}

An important experimentally accessible statistical measure is the stationary distribution of the (subthreshold) membrane

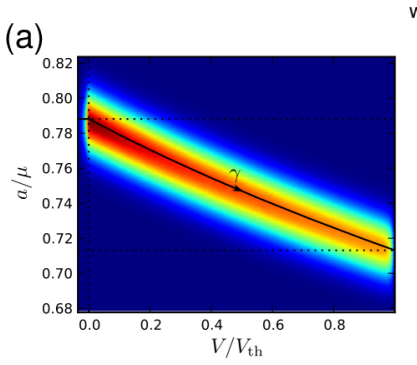

weak noise

(b)
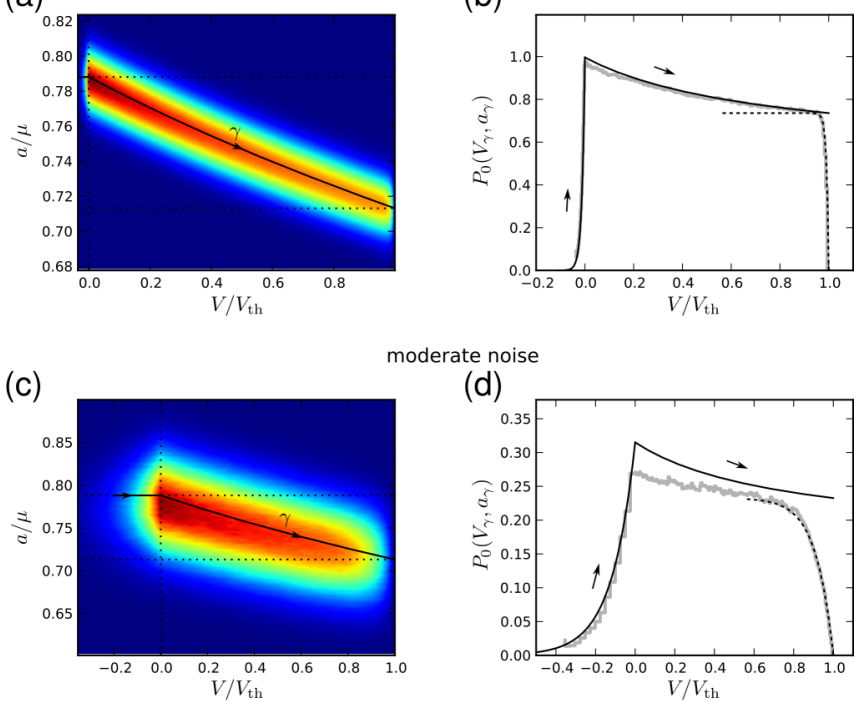

moderate noise

(d)

(e)

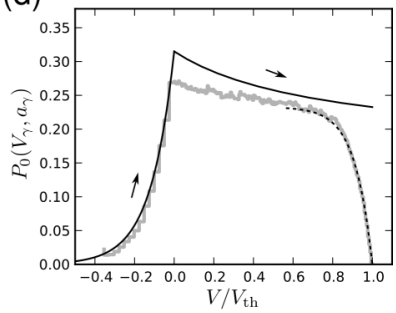

strong, fast adaptation

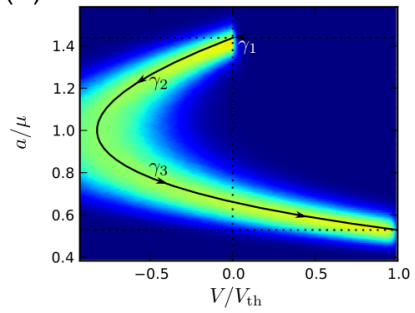

(f)

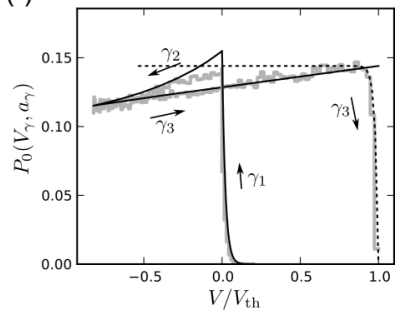

FIG. 7. (Color online) Stationary probability density $P_{0}(V, a)$. (a) $P_{0}(V, a)$ obtained from numerical simulations for weak noise $(\bar{D}=0.01)$. The solid black line indicates the limit cycle in the region $0 \leqslant V \leqslant V_{\text {th }}$, the dashed vertical line indicates the reset line, and the dashed horizontal lines indicate the locations of $a^{*}$ and $a^{*}-\Delta$. (b) A cross section of $P_{0}(V, a)$ along the solid black curve $\gamma$ is depicted by the gray line (simulation). The theory based on the WKB solution, Eq. (60), and the solution in region I, Eq. (58), is represented by the solid black line. The inner solution of the threshold layer, Eq. (59), is depicted by the dashed line. (c, d) The corresponding curves for $D=0.1$. (a-d) $\bar{\tau}_{a}=10, \bar{\Delta}=3$, for which $a^{*}<\mu$; (e, f) $\bar{D}=0.1, \bar{\tau}_{a}=1, \bar{\Delta}=10$, which corresponds to the case $a^{*}>\mu$. Arrows indicate how the stationary solution is traced out.

potential. How does adaptation shape this probability density? To this end, it is useful to compare this function to the probability density of the voltage for a PIF neuron with the same mean ISI $T^{*}$ but without adaptation current. This density is given by [43]

$$
P_{\mathrm{PIF}}(V)=\frac{1}{V_{\mathrm{th}}} \begin{cases}1-e^{\frac{V_{\mathrm{th}}}{D T^{*}}\left(V-V_{\mathrm{th}}\right)}, & 0 \leqslant V \leqslant V_{\mathrm{th}} \\ e^{\frac{V_{\mathrm{th}}}{D T^{*}}}\left(1-e^{\left.-\frac{V_{\mathrm{th}}^{2}}{D T^{*}}\right),}\right. & V<0 .\end{cases}
$$

In the case of the adapting PIF model, the marginal distribution $P_{0}(V)$ is obtained by integrating $P_{0}(V, a)$ over $a$ [cf. Eq. (29)]. For the contribution to the integral arising 
from region I, we can use our result Eq. (54):

$$
\begin{aligned}
P_{0, \mathrm{I}}(V)= & \frac{\psi_{0}\left(a^{*}\right)}{\sqrt{2 \pi \epsilon}} \exp \left(\frac{\mu-a^{*}}{\epsilon \hat{D}} V\right) \\
& \times \int_{0}^{\infty} \mathrm{d} a \exp \left(-\frac{\phi_{0}^{\prime \prime}\left(a^{*}\right)}{2 \epsilon}\left(a-a^{*}\right)^{2}\right) .
\end{aligned}
$$

For small $\epsilon$ we can extend the lower integration bound to $-\infty$, and using Eq. (51), we find

$$
P_{0, \mathrm{I}}(V)=\frac{1}{T^{*}\left|\mu-a^{*}\right|} \exp \left(\frac{\mu-a^{*}}{D} V\right) .
$$

Similarly, using the solution, Eq. (55), for the inner solution of region II, we find

$$
P_{0, \mathrm{II}, \mathrm{in}}(V)=\frac{1-\exp \left[\frac{\mu-a^{*}+\Delta}{D}\left(V-V_{\mathrm{th}}\right)\right]}{T^{*}\left(\mu-a^{*}+\Delta\right)}
$$

for $V=V_{\text {th }}-O(\epsilon)$.

In contrast, the integration of the outer solution of region II over $a$ is considerably more difficult for two reasons. First, the outer solution is known only in the parametric form, Eq. (56); i.e., the dependence on the integration variable $a$ is only implicit. Second, for the case $a^{*}>\mu$, the vertical integration path in the phase plane [Fig. 3(b)] can have zero, one, or two intersections with the limit curve $\mathcal{C}^{*}$, depending on the membrane potential $V$. For the approximation of the integral, these three cases have to be treated separately.

To overcome these difficulties, we start with the WKB solution, Eq. (43):

$$
P_{0, \text { II out }}(V)=\frac{1}{\sqrt{2 \pi \epsilon}} \int \mathrm{d} a \Psi(V, a) \exp \left[-\frac{\Phi(V, a)}{\epsilon}\right] .
$$

Because the integrand is strongly peaked about the limit cycle $\mathcal{C}^{*}$ we can employ Laplace's method (see, e.g., [40]) to obtain an approximation for this integral. The idea is to find the values $a_{\min }$ that minimize the potential $\Phi(V, a)$ for a given $V$ because these values dominate the integral, Eq. (65), asymptotically for $\epsilon \rightarrow 0$. The minima of $\Phi(V, a)$ (at a fixed $V$ ) are simply given by the intersections of the integration path with the limit curve $\mathcal{C}^{*}$. According to Laplace's method, the integration can be reduced to small neighborhoods of the minima $a_{\min }$ (if minima exist). In these neighborhoods, we can expand $\Psi(V, a)$ and $\Phi(V, a)$ to leading order:

$$
\begin{aligned}
& \Psi(V, a) \approx \Psi\left(V, a_{\min }\right), \\
& \Phi(V, a) \approx \frac{\left(a-a_{\min }\right)^{2}}{2} \partial_{a}^{2} \Phi\left(V, a_{\min }\right) .
\end{aligned}
$$

Then the integral can be approximated by the sum over all neighborhoods,

$$
P_{0, \mathrm{II}, \mathrm{out}}(V)=\sum_{a_{\min }} \frac{\Psi\left(V, a_{\min }\right)}{\sqrt{\partial_{a}^{2} \Phi\left(V, a_{\min }\right)}}=\sum_{\tau \in \tau(V)} \frac{\psi\left(\tau, a^{*}\right)}{\sqrt{\partial_{a}^{2} \phi\left(\tau, a^{*}\right)}},
$$

where the functions $\psi(\tau, s)$ and $\phi(\tau, s)$ correspond to $\Psi(V, a)$ and $\Phi(V, a)$ in characteristic coordinates. In the last step, we have used the fact that the limit curve is given by $s=a^{*}$ and $0 \leqslant \tau \leqslant T^{*}$. The sum is over the values of $\tau$ that correspond to a given value of $V$. This is provided by the inverse mapping $\tau=\tau(V)$, being the solutions of

$$
V(\tau)=\mu \tau-\tau_{a} a^{*}\left(1-e^{-\tau / \tau_{a}}\right)
$$

[cf. Eq. (44)]. The functions $\psi\left(\tau, a^{*}\right)$ and $\partial_{a}^{2} \phi\left(\tau, a^{*}\right)$ are derived in the Appendix [Eqs. (A67), (A78), and (A76)]. Because of the different numbers of possible minima, we consider the cases $a^{*}<\mu$ and $a^{*}>\mu$ separately.

\section{The case $a^{*}<\mu$}

In this case, there is only one intersection with the limit curve $\mathcal{C}^{*}$ corresponding to a single value $a_{\min }$ [Fig. 3(a)] and a unique inverse mapping $\tau(V)$ (Fig. 5). From Eq. (66) we obtain

$$
P_{0, \mathrm{II}, \mathrm{out}}(V)=\frac{1}{T^{*}\left(\mu-a^{*} e^{-\tau / \tau_{a}}\right)},
$$

which, together with Eq. (67), represents a parametric solution. This expression is equal to the inverse drift velocity $(\mu-$ $\left.a^{*} e^{-\tau / \tau_{a}}\right) T^{*}$ of a deterministic trajectory starting at $a_{i}=a^{*}$; in particular, it does not depend on the noise intensity.

A uniform approximation in region II can be achieved by adding the inner solution, Eq. (64), and the outer solution, Eq. (68), and subtracting the common limits $V \rightarrow-\infty$ for the inner solution and $\tau \rightarrow T^{*}$ for the outer solution. Together with Eq. (63), this results in the final solution

$$
P_{0}(V)= \begin{cases}\frac{1 / T^{*}}{\mu-a^{*}} \exp \left(\frac{\mu-a^{*}}{D} V\right), & V<0 ; \\ \frac{1 / T^{*}}{\mu-a^{*} e^{-\frac{\tau}{\tau a}}}-\frac{\exp \left[\frac{\mu-a^{*}+\Delta}{D}\left(V-V_{\mathrm{th}}\right)\right.}{T^{*}\left(\mu-a^{*}+\Delta\right)}, & 0 \leqslant V \leqslant V_{\mathrm{th}} .\end{cases}
$$

Apart from the parametric representation, Eqs. (67) and (69), an explicit representation is possible through the inverse relation $\tau=\tau(V)$. This relation can be expressed in terms of the upper branch $W_{0}(y)$ of the Lambert $W$ function [44]:

$$
\begin{aligned}
\tau(V) & =\tau_{+}\left(V, a^{*}\right) \\
& \equiv \tau_{a}\left[W_{0}\left(-\frac{a^{*}}{\mu} \exp \left(-\frac{V+\tau_{a} a^{*}}{\mu \tau_{a}}\right)\right)+\frac{V+\tau_{a} a^{*}}{\mu \tau_{a}}\right] .
\end{aligned}
$$

As a first check of our approximation for the probability density of the membrane potential, one can show that Eq. (69) converges to the probability density of the nonadapting PIF, Eq. (61), in two limit cases: (i) for $\tau_{a} \rightarrow \infty, \tilde{\Delta}=$ const. (with $\mu$ replaced by the effective base current $V_{\mathrm{th}} / T^{*}$ ) and (ii) for $\tilde{\Delta} \rightarrow 0, \tau_{a}=$ const. (with the same parameters $\mu$ and $D$ ).

Further confirmation of our approximation comes from the comparison to simulation results. The theory, Eq. (69), yields an excellent fit to the simulations in the limit of weak noise (cf. Fig. 8 for $\bar{D}=0.001$ and $\bar{D}=0.01$ ). The density in region II is not as flat as in the case of the nonadapting PIF model; the latter density, Eq. (61), converges to a "box," $P_{\mathrm{PIF}}(V) \rightarrow$ $V_{\text {th }}^{-1} \theta(V) \theta\left(V_{\text {th }}-V\right)$ for $D \rightarrow 0$.

The convex decrease between reset point and boundary layer reflects the above-mentioned fact that the outer solution $P_{0, \text { II out }}(V)$ is equal to the inverse of the mean drift velocity $\mu-a^{*} \exp \left(-\tau(V) / \tau_{a}\right)$. Close to the reset point the velocity is small because of the large adaptation current $a(t) \sim a^{*}$; this increases the probability in this region. As the adaptation 


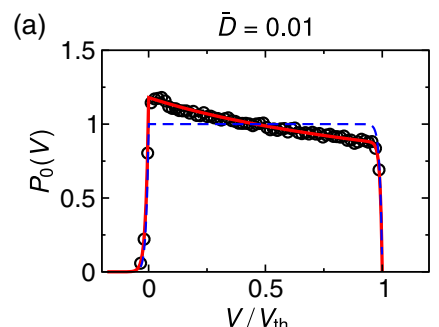

(c)

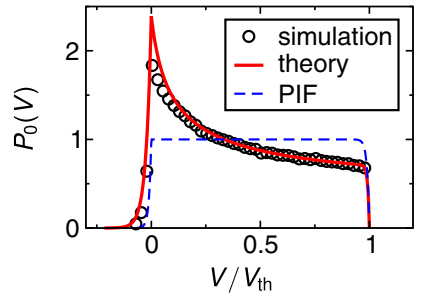

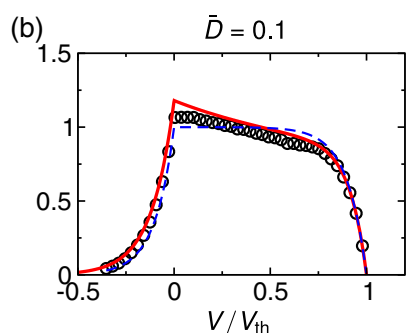

(d)

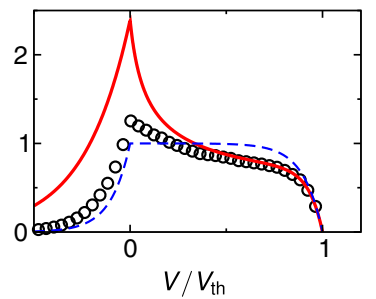

FIG. 8. (Color online) Distribution of the membrane potential in the case $a^{*}<\mu$. (a, b) Probability density $P_{0}(V)$ for slow adaptation $\bar{\tau}_{a}=10$ and different noise intensities: (a) $\bar{D}=0.01$ and (b) $\bar{D}=0.1$. The adaptation strength is $\bar{\Delta}=3$. Circles display the histograms obtained from simulations; the solid (red) line displays the weak-noise theory given by Eq. (69). The dashed (blue) line represents the probability density of the corresponding nonadapting PIF model, Eq. (61). (c, d) The corresponding figures for the case of fast adaptation, $\bar{\tau}_{a}=1, \bar{\Delta}=1$, i.e., (c) $\bar{D}=0.01$ and (d) $\bar{D}=0.1$.

current decays, $V(t)$ passes with increasing velocity towards threshold, which leads to a reduced probability at a larger membrane potential. This also explains why for a slower adaptation current the decay of the distribution is also slower [cf. Figs. 8(a) and 8(b)].

At a larger noise intensity (e.g., $\bar{D}=0.1$ in Fig. 8), the probability density is significantly overestimated by the theory. However, the pronounced decay of $P_{0}(V)$ is still in qualitative agreement with the weak-noise theory. Note that our approximation of $P_{0}(V)$ is generally not normalized; clearly, the theoretical curves for $\bar{D}=0.1$ in Figs. 8(b) and 8 (d) are for all voltage values above the simulation result, and hence, the integral is not 1 . In our theory, the normalization condition is satisfied only in the asymptotic limit of weak noise.

The theoretically predicted convex shape of the probability density outside the threshold boundary layer is a special feature induced by a fast adaptation current. This feature is not present in typical one-dimensional, white-noise-driven IF neurons that operate in the tonically firing (superthreshold) regime. In particular, the nonadapting PIF model clearly exhibits a concave voltage distribution [cf. Eq. (61)]. Note, however, that a convex membrane potential distribution can also emerge without an adaptation mechanism: For instance, this is the case for the leaky IF model with a resting potential sufficiently below the threshold (subthreshold regime), in which case the voltage distribution becomes approximately Gaussian.

\section{The case $a^{*}>\mu$}

The application of Laplace's method for the integral, (65), is more complicated because below the reset point in $V_{\text {vertex }}<$ $V \leqslant 0$ the stationary probability density now possesses two

maxima with respect to $a$ [cf. Fig. 7(e)]. The vertex $V_{\text {vertex }}$ of the limit cycle is determined by $\dot{V}=0$ or $a^{*} e^{\tau / \tau_{a}}=\mu$, implying that

$$
V_{\mathrm{vertex}}=\tau_{a}\left[\mu-a^{*}+\mu \ln \left(\frac{a^{*}}{\mu}\right)\right] .
$$

The two maxima correspond to the upper branch $\mathcal{C}_{-}^{*}$ [where $\left.a\left(\tau, a^{*}\right)>\mu\right]$ and the lower branch $\mathcal{C}_{+}^{*}$ [where $\left.a\left(\tau, a^{*}\right)<\mu\right]$, and both contribute to the integral, (65). For the lower branch we find, in leading order,

$$
P_{+}(V)=\frac{1 / T^{*}}{\left|\mu-a^{*} e^{-\frac{\tau_{+}\left(V, a^{*}\right)}{\tau_{a}}}\right|}-\frac{\exp \left[\frac{\mu-a^{*}+\Delta}{D}\left(V-V_{\mathrm{th}}\right)\right]}{T^{*}\left(\mu-a^{*}+\Delta\right)},
$$

equivalent to the explicit solution in region II for $\mu<a^{*}$ and with $\tau_{+}\left(V, a^{*}\right)$ given in Eq. (70). For the lower branch we obtain

$$
P_{-}(V)=\frac{1}{T^{*}}\left|\mu-a^{*} \exp \left(-\frac{\tau_{-}\left(V, a^{*}\right)}{\tau_{a}}\right)\right|^{-1},
$$

where $\tau_{-}\left(V, a^{*}\right)$ is given in terms of the lower branch of the Lambert $W$ function:

$$
\tau_{-}\left(V, a^{*}\right)=\tau_{a}\left[W_{-1}\left(-\frac{a^{*}}{\mu} e^{-\frac{V+\tau_{a} a^{*}}{\mu \tau_{a}}}\right)+\frac{V+\tau_{a} a^{*}}{\mu \tau_{a}}\right] .
$$

Finally, for $V>0$ the contribution from the lower branch is complemented by the probability in region I [cf. Fig. 3(b)], given by Eq. (63). Adding all parts together results in the following distribution of the membrane potential:

$$
P_{0}(V)= \begin{cases}0, & V<V_{\text {vertex }} \\ P_{+}(V)+P_{-}(V), & V_{\text {vertex }} \leqslant V<0 \\ P_{+}(V)+P_{0, \mathrm{I}}(V), & V \geqslant 0\end{cases}
$$

Close to the vertex, the maxima approach each other and the curvature $\left.\Phi_{a a}(V, a)\right|_{\mathcal{C}^{*}}$ becomes small. At the vertex, the curvature vanishes, $\Phi_{a a}\left(V_{\text {vertex }}, \mu\right)=0$, as seen from Eq. (A76) with $a^{*} e^{\tau / \tau_{a}}=\mu$. As a consequence of Eq. (66), our approximation diverges, which is obviously wrong.

The second problem associated with the divergence is that we were not able to obtain a solution for $V<V_{\text {vertex }}$ which is continuous at $V_{\text {vertex }}$. This is why in Eq. (75) we set the probability density to 0 for $V<V_{\text {vertex }}$. Apart from the behavior around $V=V_{\text {vertex }}$, the theoretical prediction, Eq. (75), agrees well with simulations if noise is sufficiently weak [cf. Figs. 9(a) and 9(d), for $\bar{D}=0.001$ ]. The probability density attains a maximum close to the vertex, i.e., close to the turning point of the negative voltage excursion. The probability for $V<0$ is large because (i) for negative values of $V$ the deterministic velocity of $V$ is small and (ii) the contributions from the two branches of the limit cycle add up. There is a rather sharp transition in the density at the reset point: here the density drops to about half the value observed just below the reset point. The two convex decays of the density (below and above the reset voltage), if observed in experiments, could thus be regarded as an indication of a strong spike-triggered adaptation current. 


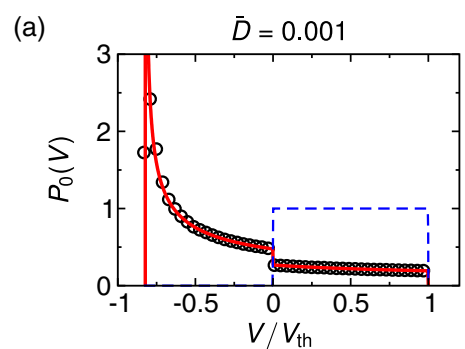

(d)

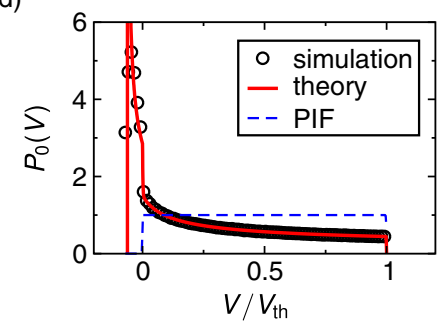

(b)

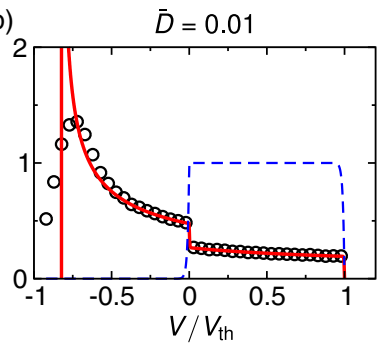

(e)

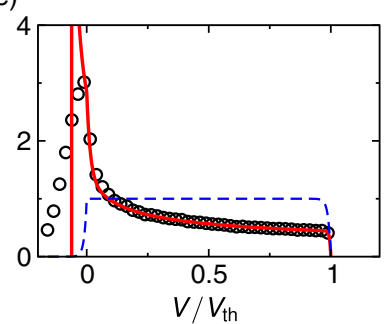

(c)

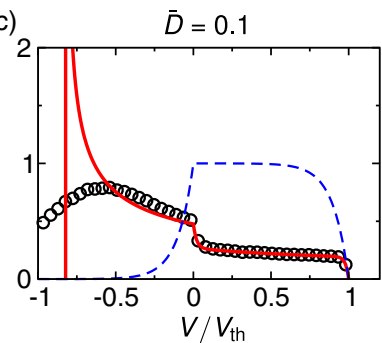

(f)

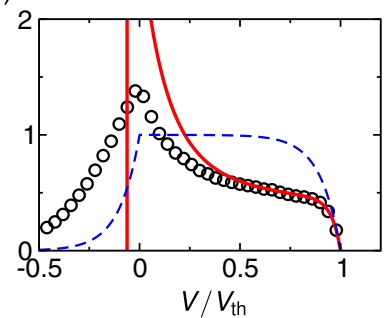

FIG. 9. (Color online) Distribution of the membrane potential for $a^{*}>\mu$. As in Fig. 8, the probability density $P_{0}(V)$ is shown for different noise intensities: (a, d) $\bar{D}=0.001$; (b, e) $\bar{D}=0.01$; (c, d) $\bar{D}=0.1$ ). Circles display the histograms obtained from simulations; the solid (red) line represents the weak-noise theory given by Eq. (75). The dashed (blue) line indicates the probability density of the corresponding nonadapting PIF model, Eq. (61). (a-c) The case of a strong adaptation current $(\bar{\Delta}=10)$, which leads to a strong negative excursion of the membrane potential [cf. Fig. 7(e)]. (d-f) The adaptation current is weaker ( $\bar{\Delta}=3)$, leading only to a small negative excursion [cf. Fig. 2(b)]. In all cases, $\bar{\tau}_{a}=1$.

\section{Distribution of the adaptation variable upon firing}

With noise, the initial value $a_{i}$ of the adaptation variable at the beginning of the $i$ th ISI becomes a stochastic variable, which scatters around $a_{i} \approx a^{*}$ with a typical variance $\sigma_{a}^{2}$. Because $a(t)$ decays exponentially afterwards $(a(t)=$ $\left.a_{i} \exp \left[-\left(t-t_{i}\right) / \tau_{a}\right]\right)$ until the voltage hits threshold, the variability of the $a_{i}$ is the only direct adaptation-induced cause of ISI variability. This is why the distribution of the initial values $a_{i}$ is particularly important.

The probability density of $a$ sampled immediately before firing, $P_{\mathrm{f} \text {,pre }}(a)$, is proportional to the efflux at the threshold:

$$
\begin{aligned}
P_{\mathrm{f}, \text { pre }}(a) & \left.\sim \partial_{V} P(V, a)\right|_{V=V_{\mathrm{th}}} \\
& \sim(\mu-a) \Psi\left(V_{\mathrm{th}}, a\right) \exp \left(-\frac{\Phi\left(V_{\mathrm{th}}, a\right)}{\epsilon}\right),
\end{aligned}
$$

which approaches a Gaussian with mean $a^{*}-\Delta$ and variance $\epsilon / \phi_{0}^{\prime \prime}\left(a^{*}\right)$ in the weak-noise limit. Consequently, in the same limit, the distribution of $a$ sampled immediately after firing, $P_{\mathrm{f}}(a)=P_{\mathrm{f}, \mathrm{pre}}(a-\Delta)$, is given by

$$
P_{\mathrm{f}}(a)=\frac{1}{\sqrt{2 \pi \sigma_{a}}} \exp \left(-\frac{\left(a-a^{*}\right)^{2}}{2 \sigma_{a}^{2}}\right),
$$

with the variance of $a_{i}$ given by

$$
\sigma_{a}^{2}=\frac{2 D a^{* 2} T^{*}\left(a^{*}-\Delta\right)^{2}}{\tau_{a}^{2} \mu \Delta\left[2 a^{*}\left(\mu-a^{*}+\Delta\right)-\mu \Delta\right]} .
$$

This approximation is in good agreement with simulations at weak noise [cf. Fig. 10(a)]. For strong noise, the distribution is slightly shifted to values of $a_{i}$ larger than predicted by the theory, although the Gaussian shape seems to be preserved [Fig. 10(b)].

In Fig. 11 we test the theoretical prediction for the standard deviation of $a_{i}$ [i.e., the square root of the variance given in
Eq. (78)] as a function of the noise intensity [Fig. 11(a)] and adaptation time constant [Fig. 11(b)] against the numerical simulations. The square root dependence on the noise intensity is confirmed over a large range of parameters and the theory is quantitatively in agreement with simulation results up to intensities of about $\bar{D}=1$. Furthermore, the dependence on $\tau_{a}$ reveals an excellent agreement for all values. This plot illustrates that our theory does not hinge on assumptions about the involved time scales, but only on the weakness of noise.

The standard deviation of the $a_{i}$ exhibits a pronounced maximum with respect to the adaptation time constant [Fig. 11(b)]. One can check quite easily that, with our scaling of the jump amplitude in the $a$ dynamics, in both limits $\tau_{a} \rightarrow 0$ and $\tau_{a} \rightarrow \infty$ the variance $\sigma_{a}^{2}$ must vanish. In the first limit any initial value decays in finite time to 0 , leaving no variability of the adaptation variable when the threshold is reached and hence no variability of the new initial values. In the second limit,
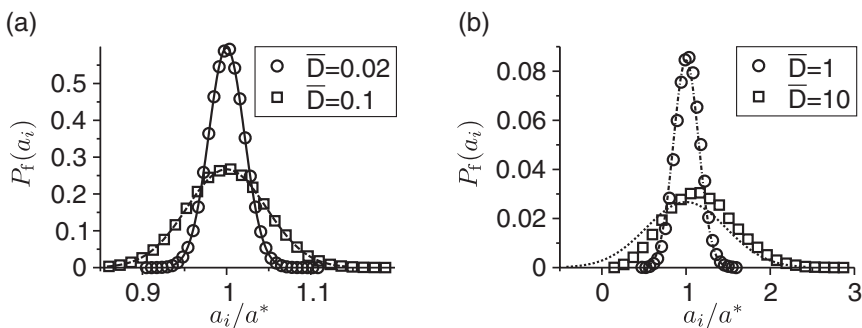

FIG. 10. Stationary probability density of the peak adaptation currents. The stationary probability density $P_{\mathrm{f}}(a)$ is shown for $\bar{\tau}_{a}=$ $10, \bar{\Delta}=3$, and different noise intensities $\bar{D}=D T^{*} / V_{\mathrm{th}}^{2}$, as indicated in the legends. Theoretical curves (solid, $\bar{D}=0.02$; dashed, $\bar{D}=0.1$; dashed-dotted, $\bar{D}=1$; dotted, $\bar{D}=10$ ) are based on the Gaussian approximation, Eq. (77). The probability density has units of $T^{*} / V_{\text {th }}$ with $T^{*}=0.1$ and $V_{\text {th }}=1$. 
(a)

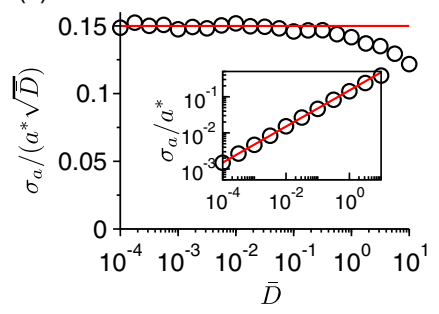

(b)

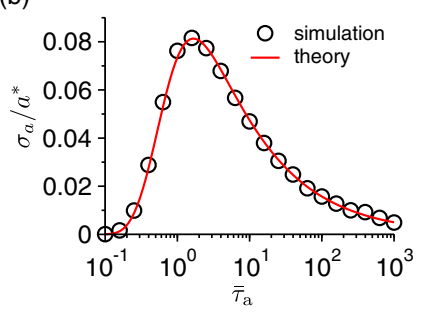

FIG. 11. (Color online) Standard deviation of the peak adaptation currents. (a) The standard deviation $\sigma_{a}$ normalized by $a^{*}$ and $\sqrt{\bar{D}}$ as a function of the noise intensity $\bar{D}=D T^{*} / V_{\mathrm{th}}^{2}$. Inset: $\sigma_{a}$ normalized by $a^{*}$ as a function of $\bar{D}$ in a double-logarithmic plot. Parameters: $\bar{\tau}_{a}=$ 10, $\bar{\Delta}=3$. (b) Normalized standard deviation $\sigma_{a} / a^{*}$ as a function of the adaptation time constant $\bar{\tau}_{a}=\tau_{a} / T^{*}$. Parameters: $\bar{D}=0.1$, $\bar{\Delta}=3$. In both panels, solid lines depict the theory, Eq. (78), and circles represent simulations.

of infinitely large adaptation time, the jump size $\Delta=\tilde{\Delta} / \tau_{a}$ vanishes, which leads once more to vanishing variance of the adaptation variable. From the two limit cases we can conclude that $\sigma_{a}\left(\tau_{a}\right) / a^{*}$ must display at least one maximum.

\section{SUMMARY}

In this paper we have calculated an approximation for the stationary probability density of an adapting PIF model. This solution is valid for weak noise but does not require the common time-scale separation arguments of slow adaptation. More generally, it is one of the rare cases for which the steadystate density can be calculated for a two-dimensional stochastic system that does not obey detailed balance (for other examples, see [2] and [45]). It is conceivable that the method developed in our paper can also be applied to other two-dimensional systems [46] with simple piecewise constant or linear drift terms, e.g., the piecewise-linear FitzHugh-Nagumo model [47-49]. In each stripe of the phase space with continuous dynamics, the stationary probability density can be approximately calculated close to a one-dimensional manifold using a WKB ansatz if the noise is weak or the time-scale separation between slow and fast variables is large. Similarly to the present model, the probability density and the local curvature of the nonequilibrium potential on the one-dimensional limit curve then have to be matched at the interfaces of neighboring stripes.

Most interestingly in the neurobiological context are two findings that are well described by our theory. The first is that the marginal probability density of the voltage attains a particular shape due to the presence of adaptation. Although adaptation currents become apparent first in the spike-train statistics, the strongly convex shape of the membrane potential probability density can be regarded as another indicator of spike-triggered adaptation. It remains to be investigated, however, whether subthreshold nonlinearities, which were neglected in our model, can have a similar effect on the probability density. If the resting potential $\mu$ is close to or above the threshold, a simple leak term does not seem to suffice to evoke a strongly convex shape of $P_{0}(V)$ (see Fig. 2.5 in [50]).
Our second finding for the PIF model with adaptation concerns the width of the distribution of the adaptation variable upon firing. This density has special meaning because it quantifies the amount of fluctuations that the adaptation variable contributes to the variability of the ISI. Note that in our model with deterministic adaptation dynamics, fluctuations in the adaptation variable upon firing originate entirely in the variability of previous ISIs. Dynamical noise, as it may emerge in the dynamics of $a(t)$ through channel noise $[27,35]$, would contribute additional variability to the ISI. For our model we have shown that the variance of the adaptation variable upon firing passes through a maximum as a function of the adaptation time constant. The maximal effect of adaptation variability is thus not observed in the slow-adaptation time limit but rather in the case in which the mean ISI and adaptation constant are of comparable orders of magnitude.

An interesting problem for future research is to inspect the response of the model to an external signal. As known for one-dimensional IF models [51], the steady-state distribution that we have calculated here could be the starting point for the linear response calculation with respect to a weak external signal. Importantly, our framework is not restricted to slow adaptation approximations of the linear response function [23,39]. This could help us to understand from an analytical point of view aspects of signal transmissions in adapting neuron models that have been observed in numerical simulations of conductance-based model neurons [18].

\section{ACKNOWLEDGMENTS}

This research project was funded by the BMBF (FKZ: 01GQ1001A) and by the European Research Council under Grant Agreement No. 268689 (MultiRules).

\section{APPENDIX: SOLUTION OF THE STATIONARY FOKKER-PLANCK EQUATION}

Here, a detailed derivation of the stationary solution $P_{0}(V, a)$ is given. The solution satisfies the boundary value problem

$$
-(\mu-a) \partial_{V} P_{0}+\frac{1}{\tau_{a}} \partial_{a}\left(a P_{0}\right)+\epsilon \hat{D} \partial_{V}^{2} P_{0}=0
$$

with the boundary conditions

$$
\begin{gathered}
P_{0}\left(V_{\mathrm{th}}, a\right)=0, \\
P_{0}\left(0^{-}, a\right)=P_{0}\left(0^{+}, a\right), \\
\partial_{V} P_{0}\left(0^{+}, a\right)-\partial_{V} P_{0}\left(0^{-}, a\right)=\partial_{V} P_{0}\left(V_{\mathrm{th}}, a-\Delta\right), \\
\lim _{V \rightarrow-\infty} P_{0}(V, a)=0, \quad \lim _{a \rightarrow \infty} P_{0}(V, a)=0, \\
-\epsilon \hat{D} \int_{0}^{\infty} \mathrm{d} a \partial_{V} P_{0}\left(V_{\mathrm{th}}, a\right)=\frac{1}{T^{*}} .
\end{gathered}
$$

We seek the solution in different regions as depicted in Fig. 3. These partial solutions are then patched together according to the boundary conditions. 


\section{Region I}

We first consider region I, which is defined by $V<0$ in the case $a^{*}<\mu$ and by $V>0$ and $a>\mu$ in the case $a^{*}>\mu$. Taking the direct limit $\epsilon \rightarrow 0$ of Eq. (A1) yields

$$
-(\mu-a) \partial_{V} P_{0}+\frac{1}{\tau_{a}} \partial_{a}\left(a P_{0}\right)=0 .
$$

This equation is valid if the third term $\epsilon \hat{D} \partial_{V}^{2} P_{0}$ vanishes in limit $\epsilon \rightarrow 0$. The only solution of Eq. (A7) that satisfies the boundary condition, (A5), is the trivial solution $P_{0}(V, a)=0$. However, this solution cannot be reconciled with the continuity condition, (A3), at $V=0$ because the left-side limit $P_{0}\left(0^{+}, a\right)$ is finite, in general. This problem can be resolved by using boundary layer theory [40]. In fact, the true solution develops a boundary layer, $-\epsilon<V \leqslant 0$, in which $P_{0}(V, a)$ rises steeply from $P_{0}(V, a)=0$ to $P_{0}\left(0^{+}, a\right)>0$ as $V \rightarrow 0-$ (Fig. 4). Because of the rapid change in $P_{0}$ within the boundary layer, the diffusion term $\epsilon \hat{D} \partial_{V}^{2} P_{0}$ cannot be neglected versus the first two terms in Eq. (A1) even for vanishing $\epsilon$. To obtain the leading-order solution in the boundary layer (the so-called "inner solution"), we introduce the stretched variable $x=V / \epsilon$. Using the rescaled function $\hat{P}_{0}(x, a)=\sqrt{\epsilon} P_{0}(V, a)$, the Fokker-Planck equation reads

$$
-(\mu-a) \partial_{x} \hat{P}_{0}+\frac{\epsilon}{\tau_{a}} \partial_{a}\left(a \hat{P}_{0}\right)+\hat{D} \partial_{x}^{2} \hat{P}_{0}=0 .
$$

In leading order, the second term can be dropped, resulting in the ordinary differential equation

$$
-(\mu-a) \partial_{x} \hat{P}_{0}+\hat{D} \partial_{x}^{2} \hat{P}_{0}=0,
$$

subjected to the boundary conditions

$$
\begin{gathered}
\lim _{x \rightarrow-\infty} \hat{P}_{0}(x, a)=0, \\
\hat{P}_{0}\left(0^{-}, a\right)=\hat{P}_{0}\left(0^{+}, a\right) .
\end{gathered}
$$

The solution of Eq. (A9) that respects (A10) is

$$
\hat{P}_{0}(x, a)=\frac{M(a)}{\lambda(a)} e^{\lambda(a) x},
$$

where $\lambda(a)=(\mu-a) / \hat{D}$. The function $M(a)$ is determined by the continuity condition, (A11). Returning to the variable $V$, the leading-order solution in region $\mathrm{I}$ is

$$
P_{0}(V, a)=\frac{M(a)}{\sqrt{\epsilon} \lambda(a)} \exp \left(\frac{\lambda(a) V}{\epsilon}\right) .
$$

\section{Region II}

In region II (see Fig. 3), the solution is sought in the vicinity of the limit curve $\mathcal{C}^{*}$. In the deterministic limit, the probability density is singular, with all probability concentrated in this limit set. For weak but finite noise, the probability density will be smeared out, with a nonsingular, Gaussian-like profile around $\mathcal{C}^{*}$. To obtain this profile, we use the WKB ansatz [41],

$$
P_{0}(V, a)=\mathcal{N}(\epsilon) \Psi(V, a) \exp \left(-\frac{\Phi(V, a)}{\epsilon}\right) .
$$

The functions $\Phi(V, a)$ and $\Psi(V, a)$ are independent of $\epsilon$ and the normalization factor must be proportional to $1 / \sqrt{\epsilon}$ (see Appendix, Sec. A 4). Specifically it is chosen as

$$
\mathcal{N}(\epsilon)=\frac{1}{\sqrt{2 \pi \epsilon}} .
$$

Inserting the ansatz, (A14), into the Fokker-Planck equation, (A1), gives

$$
\begin{aligned}
0= & {\left[(\mu-a) \Phi_{V}-\frac{a}{\tau_{a}} \Phi_{a}+\hat{D} \Phi_{V}^{2}\right] \Psi } \\
& +\epsilon\left[-(\mu-a) \Psi_{V}+\frac{a}{\tau_{a}} \Psi_{a}+\frac{1}{\tau_{a}} \Psi\right. \\
& \left.-2 \hat{D} \Phi_{V} \Psi_{V}-\hat{D} \Phi_{V V} \Psi\right]+\epsilon^{2} \Psi_{V V} .
\end{aligned}
$$

The indices on $\Phi$ and $\Psi$ are shorthand notations for the respective partial derivatives, e.g., $\Phi_{V}=\partial_{V} \Phi, \Phi_{V V}=\partial_{V}^{2} \Phi$. The terms inside the brackets must be 0 , hence

$$
\begin{gathered}
(\mu-a) \Phi_{V}-\frac{a}{\tau_{a}} \Phi_{a}+\hat{D} \Phi_{V}^{2}=0, \\
\left(\mu-a+2 \hat{D} \Phi_{V}\right) \Psi_{V}-\frac{a}{\tau_{a}} \Psi_{a}=\left(\frac{1}{\tau_{a}}-\hat{D} \Phi_{V V}\right) \Psi .
\end{gathered}
$$

Equation (A17) is a first-order, nonlinear partial differential equation for $\Phi(V, a)$ and independent of $\Psi(V, a)$. Inserting the solution into Eq. (A18) yields a closed, linear, first-order equation for $\Psi(V, a)$. In the context of WKB theory, Eqs. (A17) and (A18) are known as the eikonal and transport equations, respectively.

In general, the solutions $\Phi$ and $\Psi$ cannot satisfy both boundary conditions at $V=0$ and $V=V_{\text {th }}$ because they obey first-order differential equations. This again indicates a boundary layer structure of the solution: The WKB solution, (A14), that satisfies the boundary condition at $V=0$ is valid in region II except for a thin boundary layer of thickness $O(\epsilon)$ close to the threshold [Fig. 4(b)]. In this boundary layer, the actual solution drops rapidly to 0 because of the absorbing boundary. To obtain an approximate solution that fulfills both boundary conditions, the solutions outside the boundary layer $\left[V=V_{\text {th }}-O(1)\right]$ and inside the boundary layer $\left[V=V_{\text {th }}-O(\epsilon)\right]$ are first calculated separately ("outer" and "inner" solutions). We then use the method of asymptotic matching to connect the solutions in the overlap region $V=V_{\mathrm{th}}-O(\sqrt{\epsilon})$.

\section{a. WKB solution}

The boundary conditions of Eqs. (A17) and (A18) at $V=0$ are given by the functions

$$
\Phi(0, a)=\phi_{0}(a)
$$

and

$$
\Psi(0, a)=\psi_{0}(a)
$$

which are determined later using the reset condition. There are further constraints of the WKB solution evaluated on the limit 
cycle: Because for $\epsilon=0$ all probability is concentrated on the limit curve $\mathcal{C}^{*}, \Phi$ must vanish on $\mathcal{C}^{*}$ :

$$
\left.\Phi(V, a)\right|_{\mathcal{C}^{*}}=0 .
$$

Furthermore, the probability density should not diverge in the neighborhood of $\mathcal{C}^{*}$ as $\epsilon$ tends to 0 . Hence, $\Phi$ must be locally non-negative. This is ensured by the conditions

$$
\left.\Phi_{V}(V, a)\right|_{\mathcal{C}^{*}}=0,\left.\quad \Phi_{a}(V, a)\right|_{\mathcal{C}^{*}}=0
$$

and

$$
\left.\Phi_{V V}(V, a)\right|_{\mathcal{C}^{*}}>0,\left.\quad \Phi_{a a}(V, a)\right|_{\mathcal{C}^{*}}>0 .
$$

Equation (A17) can be solved by the method of characteristics. This means that the solution is sought in a coordinate system, in which the partial differential equation, (A17), becomes an ordinary differential equation. To this end, $V$, $a$, and $\Phi$, as well as its partial derivatives $\Phi_{V}$ and $\Phi_{a}$, are regarded as functions of the characteristic coordinates $\tau$ and $s$. To make this dependence explicit, we introduce the functions

$$
\begin{gathered}
\phi(\tau, s)=\Phi(V(\tau, s), a(\tau, s)), \\
p(\tau, s)=\Phi_{V}(V(\tau, s), a(\tau, s)), \\
q(\tau, s)=\Phi_{a}(V(\tau, s), a(\tau, s)) .
\end{gathered}
$$

The variable transformation $V=V(\tau, s)$ and $a=a(\tau, s)$ can be found as follows: We require that $\tau=0$ corresponds to the reset line, which we parametrize by the coordinate $s$ :

$$
V(0, s)=0, \quad a(0, s)=s, \quad s>0 .
$$

Thus, the coordinate $s$ equals $a$ on the reset line (Fig. 5). The boundary condition, Eq. (A19), at $V=0$ becomes

$$
\phi(0, s)=\phi_{0}(s),
$$

whereas the constraints, Eqs. (A21)-(A23), give rise to the initial conditions

$$
\begin{gathered}
\phi\left(0, a^{*}\right)=0, \\
p\left(0, a^{*}\right)=0, \\
q\left(0, a^{*}\right)=0, \\
q_{a}\left(0, a^{*}\right)>0, \quad p_{V}\left(0, a^{*}\right)>0 .
\end{gathered}
$$

Equation (A17) can be rewritten in terms of $p$ and $q$ as

$$
F(a, p, q) \equiv(\mu-a) p-\frac{a}{\tau_{a}} q+\hat{D} p^{2}=0 .
$$

From the theory of first-order partial differential equations it is known that the partial derivatives $p$ and $q$ obey quasilinear first-order partial differential equations, which are found as follows: Differentiating Eq. (A33) with respect to $V$ yields

$$
F_{p} p_{V}+F_{q} p_{a}=0,
$$

where we have used $q_{V}=\phi_{a V}=p_{a}$. Analogously, differentiating Eq. (A33) with respect to $a$ and using $p_{a}=\phi_{V a}=q_{V}$ gives

$$
F_{p} q_{V}+F_{q} q_{a}=-F_{a} .
$$

The quasilinear equations, (A34) and (A35), are solved by the characteristic equations

$$
\begin{gathered}
\frac{\mathrm{d} V}{\mathrm{~d} \tau}=F_{p}=\mu-a+2 \hat{D} p, \\
\frac{\mathrm{d} a}{\mathrm{~d} \tau}=F_{q}=-\frac{a}{\tau_{a}}, \\
\frac{\mathrm{d} p}{\mathrm{~d} \tau}=0, \\
\frac{\mathrm{d} q}{\mathrm{~d} \tau}=-F_{a}=p+\frac{1}{\tau_{a}} q .
\end{gathered}
$$

The initial conditions $p(0, s) \equiv p_{0}(s)$ and $q(0, s) \equiv q_{0}(s)$ can be derived from $\phi_{0}(s)$

$$
q_{0}(s)=\Phi_{a}(0, a)=\partial_{s} \phi(0, s)=\phi_{0}^{\prime}(s) .
$$

Furthermore, Eq. (A33) evaluated at $\tau=0$ yields

$$
p_{0}^{2}+\frac{\mu-s}{\hat{D}} p_{0}-\frac{s}{\hat{D} \tau_{a}} q_{0}=0 .
$$

The quadratic equation has two solutions, one of which satisfies the constraints at $s=a^{*}$. From Eqs. (A30) and (A31) it is known that $p_{0}\left(a^{*}\right)=0$ and $q_{0}\left(a^{*}\right)=\phi^{\prime}\left(a^{*}\right)=0$. Thus, the only solution is

$$
p_{0}(s)=-\frac{\mu-s}{2 \hat{D}}+S \sqrt{\frac{(\mu-s)^{2}}{4 \hat{D}^{2}}+\frac{s}{\hat{D} \tau_{a}} \phi_{0}^{\prime}(s)},
$$

where $S=\operatorname{sgn}\left(\mu-a^{*}\right)$ is the sign of $\mu-a^{*}$.

Equation (A38) states that $p$ is conserved along the characteristics, hence

$$
p(\tau, s)=p_{0}(s)
$$

Furthermore, the solutions of Eqs. (A36), (A37), and (A39) are

$$
\begin{gathered}
V(\tau, s)=\left[\mu+2 \hat{D} p_{0}(s)\right] \tau-\tau_{a} s\left(1-e^{-\tau / \tau_{a}}\right), \\
a(\tau, s)=s e^{-\tau / \tau_{a}}, \\
q(\tau, s)=\phi_{0}^{\prime}(s) e^{\tau / \tau_{a}}-\tau_{a} p_{0}(s)\left(1-e^{\tau / \tau_{a}}\right) .
\end{gathered}
$$

A differential equation for $\phi(\tau, s)$ can be obtained by taking the total derivative of $\Phi(V, a)$ with respect to $\tau$. Using Eqs. (A36) and (A37) this yields

$$
\begin{aligned}
\frac{\mathrm{d} \phi}{\mathrm{d} \tau} & =\frac{\mathrm{d} \Phi}{\mathrm{d} \tau}=\Phi_{V} \frac{\mathrm{d} V}{\mathrm{~d} \tau}+\Phi_{a} \frac{\mathrm{d} a}{\mathrm{~d} \tau} \\
& =p F_{p}+q F_{q}=p(\mu-a)+2 \hat{D} p^{2}-\frac{a}{\tau_{a}} q=\hat{D} p^{2},
\end{aligned}
$$

where relation (A33) was used for the last equality. The parametric solution reads

$$
\phi(\tau, s)=\hat{D} p_{0}^{2}(s) \tau+\phi_{0}(s), \quad \tau, s>0 .
$$

The characteristic curves $\mathcal{C}(s)=\{(V, a, \phi)(t)\}$ with the initial conditions $V(0, s)=0, a(0, s)=s$, and $\phi(0, s)=\phi_{0}(s)$ are uniquely characterized by the parameter $s$. The union of all characteristics forms a surface in the $V, a, \Phi$ space, which represents the solution $\Phi(V, a)$. 
We can now solve Eq. (A18) for $\Psi(V, a)$. This quasilinear equation is equivalent to the characteristic equations

$$
\begin{gathered}
\frac{\mathrm{d} V}{\mathrm{~d} \tau}=\mu-a+2 \hat{D} p(s), \\
\frac{\mathrm{d} a}{\mathrm{~d} \tau}=-\frac{a}{\tau_{a}}, \\
\frac{\mathrm{d} \psi}{\mathrm{d} \tau}=\left(\frac{1}{\tau_{a}}-\hat{D} \phi_{V V}(\tau, s)\right) \psi .
\end{gathered}
$$

The solution for $V$ and $a$ is identical to Eqs. (A44) and (A45). The integration of Eq. (A50) yields

$$
\psi(\tau, s)=\psi_{0}(s) \exp \left[-\int_{0}^{\tau} \mathrm{d} t^{\prime}\left(\hat{D} \phi_{V V}\left(t^{\prime}, s\right)-\frac{1}{\tau_{a}}\right)\right] .
$$

The function $\phi_{V V}$ can be obtained using the chain rule:

$$
\begin{aligned}
\phi_{V V}(\tau, s) & =\left(\frac{\partial p}{\partial V}\right)_{a}=\frac{\partial p}{\partial \tau}\left(\frac{\partial \tau}{\partial V}\right)_{a}+\frac{\partial p}{\partial s}\left(\frac{\partial s}{\partial V}\right)_{a} \\
& =p_{0}^{\prime}(s)\left(\frac{\partial s}{\partial V}\right)_{a} .
\end{aligned}
$$

Here, $(\partial f / \partial x)_{y}$ denotes the partial derivative of $f$ with respect to $x$ with $y$ fixed. This notation also indicates that $f$ is regarded as a function of $x$ and $y$. Let us regard $V$ as a function of $s$ and a:

$$
V=V(s, a)=\left[\mu+2 \hat{D} p_{0}(s)\right] \tau_{a} \ln \frac{s}{a}-\tau_{a} s+\tau_{a} a .
$$

Differentiating this relation with respect to $V$ yields $1=$ $\left(\frac{\partial V}{\partial s}\right)_{a}\left(\frac{\partial s}{\partial V}\right)_{a}$ or

$$
\begin{aligned}
\left(\frac{\partial s}{\partial V}\right)_{a} & =\frac{1}{\left(\frac{\partial V}{\partial s}\right)_{a}} \\
& =\frac{1}{2 \hat{D} p_{0}^{\prime}(s) \tau_{a} \ln \left(\frac{s}{a}\right)+\frac{\tau_{a}}{s}\left(\mu+2 \hat{D} p_{0}(s)-s\right)} .
\end{aligned}
$$

Thus, we obtain

$$
\phi_{V V}(\tau, s)=\frac{p_{0}^{\prime}(s)}{2 \hat{D} p_{0}^{\prime}(s) \tau+\frac{\tau_{a}}{s}\left(\mu+2 \hat{D} p_{0}(s)-s\right)}
$$

and

$$
\psi(\tau, s)=\psi_{0}(s) e^{\tau / \tau_{a}} \sqrt{\frac{\mu-s+2 \hat{D} p_{0}(s)}{2 \hat{D} p_{0}^{\prime}(s) s \frac{\tau}{\tau_{a}}+\mu-s+2 \hat{D} p_{0}(s)}},
$$

which represents the solution of $\Psi(V, a)$ in parametric form.

\section{b. Inner solution and asymptotic matching with the WKB solution}

To treat the rapid change in the solution near the threshold, we pass to the stretched variable $x=\left(V-V_{\text {th }}\right) / \epsilon$. As before, the function $\hat{P}_{0}(x, a)=\sqrt{\epsilon} P_{0}(V, a)$ satisfies Eq. (A8). The leading-order solution that vanishes at the threshold, $x=0$, is given by

$$
\hat{P}_{0}(x, a)=\frac{N(a)}{\lambda(a)}\left(e^{\lambda(a) x}-1\right),
$$

where $\lambda(a)=(\mu-a) / \hat{D}$. The exponential term in Eq. (A56) shows that the thickness of the boundary layer is $d(\epsilon)=$ $\epsilon / \lambda(a)=O(\epsilon)$ as $\epsilon \rightarrow 0$. Thus, the WKB solution is a valid asymptotic approximation only for $0<V<V_{\text {th }}-\mathcal{O}(\epsilon)$ (Fig. 4). On the other hand, we assume that the inner solution is valid not only inside the boundary layer [i.e., $V=V_{\text {th }}-\mathcal{O}(\epsilon)$ ], but also at $V=V_{\text {th }}-\mathcal{O}\left(\epsilon^{1 / 2}\right)$. At this position both the WKB and the inner solution are valid and should match asymptotically. In the intermediate limit,

$$
\epsilon \rightarrow 0, \quad V \rightarrow V_{\mathrm{th}}, \quad x=\frac{V-V_{\mathrm{th}}}{\epsilon} \rightarrow-\infty,
$$

the outer solution, Eq. (A14), is asymptotic to

$$
\frac{1}{\sqrt{2 \pi \epsilon}} \Psi\left(V_{\mathrm{th}}, a\right) \exp \left(-\frac{\Phi\left(V_{\mathrm{th}}, a\right)}{\epsilon}\right) .
$$

The inner solution, Eq. (A56), converges to $-N(a) /[\sqrt{\epsilon} \lambda(a)]$. Equating both limits yields

$$
N(a)=-\frac{\lambda(a)}{\sqrt{2 \pi}} \Psi\left(V_{\mathrm{th}}, a\right) \exp \left(-\frac{\Phi\left(V_{\mathrm{th}}, a\right)}{\epsilon}\right) .
$$

\section{c. Patching regions I and II}

The still undetermined function $M(a)$ can now be specified by requiring the continuity of the probability density at $V=0$ [boundary condition Eq. (A11)]. Equations (A13) and (A14) result in

$$
M(a)=\frac{\mu-a}{\sqrt{2 \pi} \hat{D}} \Psi(0, a) \exp \left[-\frac{\Phi(0, a)}{\epsilon}\right] .
$$

\section{Reset condition}

The functions $\phi_{0}(a)=\Phi(0, a)$ and $\psi_{0}(a)=\Psi(0, a)$ are determined by the reset condition Eq. (A4) and the normalization condition Eq. (A6). Unfortunately, to analytically satisfy the reset condition for all $a>0$ seems to be rather difficult. However, in the case of weak noise, the knowledge of $\phi_{0}(a)$ and $\psi(a)$ is not necessary for all $a>1$. Rather, it is sufficient to know the local behavior in the neighborhood of $a^{*}$ because most probability is located close to the deterministic limit cycle. Using this fact, we expand $\phi_{0}(a)$ and $\psi_{0}(a)$ about $a^{*}$ to lowest order in $\delta a=a-a^{*}$ :

$$
\begin{gathered}
\phi_{0}(a)=\frac{\phi_{0}^{\prime \prime}\left(a^{*}\right)}{2} \delta a^{2}+O\left(\delta a^{3}\right), \\
\psi_{0}(a)=\psi_{0}\left(a^{*}\right)+O(\delta a),
\end{gathered}
$$

Here, we have taken into account that both $\phi_{0}(a)$ and $\phi_{0}^{\prime}(a)$ vanish on the limit-cycle (cf. equations, (A29) and (A31)); i.e., $\phi_{0}(a)$ is locally a parabola. The reset condition is now used only locally to determine $\phi_{0}^{\prime \prime}\left(a^{*}\right)$ instead of the whole function $\phi_{0}(a)$. Likewise, the normalization condition is used to calculate $\psi_{0}\left(a^{*}\right)$ (Sec. A 4$)$.

The derivatives that appear in the reset condition, Eq. (A4), can be found from the solutions, Eqs. (A13), (A14), and (A56), 
in the respective regions. For $a^{*}<\mu$, they are given by

$$
\begin{aligned}
\partial_{V} P_{0}\left(0^{+}, a\right)= & \frac{1}{\sqrt{2 \pi \epsilon^{3}}}\left(\epsilon \Psi_{V}(0, a)-\Phi_{V}(0, a) \psi_{0}(a)\right) \\
& \times \exp \left(-\frac{\phi_{0}(a)}{\epsilon}\right), \\
\partial_{V} P_{0}\left(0^{-}, a\right)= & \epsilon^{-3 / 2} M(a) \\
= & \frac{\mu-a}{\sqrt{2 \pi \epsilon^{3}} \hat{D}} \psi_{0}(a) \exp \left(-\frac{\phi_{0}(a)}{\epsilon}\right), \\
\partial_{V} P_{0}\left(V_{\mathrm{th}}, a-\Delta\right)= & \epsilon^{-3 / 2} N(a-\Delta) \\
= & -\frac{\mu-a+\Delta}{\sqrt{2 \pi \epsilon^{3}} \hat{D}} \Psi\left(V_{\mathrm{th}}, a-\Delta\right) \\
& \times \exp \left(-\frac{\Phi\left(V_{\mathrm{th}}, a-\Delta\right)}{\epsilon}\right) .
\end{aligned}
$$

In the case $a^{*}>\mu$, the expressions for $\partial_{V} P_{0}\left(0^{+}, a\right)$ and $\partial_{V} P_{0}\left(0^{-}, a\right)$ have to be interchanged. The reset condition, (A4), can now be rewritten in terms of the functions $\Phi$ and $\Psi$,

$$
\begin{aligned}
& (\mu-a+\Delta) \Psi\left(V_{\mathrm{th}}, a-\Delta\right) \exp \left(-\frac{\Phi\left(V_{\mathrm{th}}, a-\Delta\right)}{\epsilon}\right) \\
& =\operatorname{sgn}\left(\mu-a^{*}\right)\left(\mu-a+\hat{D} \Phi_{V}(0, a)\right) \psi_{0}(a) \exp \left(-\frac{\phi_{0}(a)}{\epsilon}\right),
\end{aligned}
$$

where we have neglected the higher-order term $\epsilon \Psi_{V}(0, a)$.

\section{a. Local reset condition}

As mentioned above, we only demand that the reset condition, Eq. (A62), is satisfied locally around $a=a^{*}$, i.e., close to the deterministic limit cycle. This approach is justified for weak noise. To this end, we expand both sides of Eq. (A62) about $a^{*}$ and use the properties Eqs. (A21) and (A22) for $\Phi(V, a)$ on the limit cycle. In particular, $\Phi\left(V_{\mathrm{th}}, a^{*}-\Delta\right)=$ $\phi_{0}\left(a^{*}\right)=0$ and $\Phi_{a}\left(V_{\mathrm{th}}, a^{*}-\Delta\right)=\Phi_{a}\left(0, a^{*}\right)=0$. As a result, the right-hand side of Eq. (A62) reads, up to first order in $\delta a=a-a^{*}$,

$$
\begin{aligned}
& \left(\mu-a^{*}+\Delta\right) \Psi\left(V_{\mathrm{th}}, a^{*}-\Delta\right) \\
& \quad+\delta a\left[\left(\mu-a^{*}+\Delta\right) \Psi_{a}\left(V_{\mathrm{th}}, a^{*}-\Delta\right)-\Psi\left(V_{\mathrm{th}}, a^{*}-\Delta\right)\right] .
\end{aligned}
$$

The left-hand side reads

$$
\begin{aligned}
& \left|\mu-a^{*}\right| \psi_{0}\left(a^{*}\right)+\operatorname{sgn}\left(\mu-a^{*}\right) \delta a \\
& \quad \times\left[\left(\mu-a^{*}\right) \Psi_{a}\left(0, a^{*}\right)-\left(\hat{D} \partial_{a} \Phi_{V}\left(0, a^{*}\right)-1\right) \psi_{0}\left(a^{*}\right)\right] .
\end{aligned}
$$

Equating the zeroth-order terms yields the condition

$$
\left(\mu-a^{*}+\Delta\right) \Psi\left(V_{\mathrm{th}}, a^{*}-\Delta\right)=\left|\mu-a^{*}\right| \psi_{0}\left(a^{*}\right)
$$

or, equivalently,

$$
\psi\left(T^{*}, a^{*}\right)=\frac{\left|\mu-a^{*}\right|}{\mu-a^{*}+\Delta} \psi_{0}\left(a^{*}\right) .
$$

To obtain the leading order of the stationary probability density it is sufficient to compute $\phi_{0}^{\prime \prime}\left(a^{*}\right)$ and $\psi_{0}\left(a^{*}\right)$. These quantities are completely determined by the zeroth-order condition (A66) and the normalization condition (see below). We note that, in principle, matching the first-order terms in Eqs. (A63) and (A64) could be used to obtain $\psi_{0}^{\prime}\left(a^{*}\right)$ and, hence, the firstorder term in the expansion, Eq. (A60), of the shaping factor $\psi_{0}(s)=\Psi(0, a)$. Taking into account this linear approximation of $\psi_{0}(s)$, we found that it was possible to capture the deviation of $P(0, a)$ from a Gaussian distribution as quantified by the skewness. This higher-order correction is, however, beyond the scope of this paper.

\section{b. Calculation of $\phi_{0}^{\prime \prime}\left(a^{*}\right)$}

From the full solution of $\psi(\tau, s)$, Eq. (A55), we also find

$$
\psi\left(\tau, a^{*}\right)=\frac{\psi_{0}\left(a^{*}\right) e^{\tau / \tau_{a}}}{\sqrt{\nu \tau+1}}
$$

with

$$
v=\frac{2 \hat{D} a^{* 2} \phi_{0}^{\prime \prime}\left(a^{*}\right)}{\tau_{a}^{2}\left(\mu-a^{*}\right)^{2}} .
$$

Here, we have used $p_{0}\left(a^{*}\right)=0$ and the derivative of $p_{0}(s)$ at $s=a^{*}$,

$$
p_{0}^{\prime}\left(a^{*}\right)=\frac{a^{*} \phi_{0}^{\prime \prime}\left(a^{*}\right)}{\tau_{a}\left(\mu-a^{*}\right)}
$$

[cf. Eq. (A42)]. Inserting Eq. (A67) with $\tau=T^{*}$ into Eqs. (A66) and taking into account that $e^{T^{*} / \tau_{a}}=a^{*} /\left(a^{*}-\Delta\right)$ yields

$$
v=\frac{1}{T^{*}}\left[\left(\frac{\left(\mu-a^{*}+\Delta\right) a^{*}}{\left(\mu-a^{*}\right)\left(a^{*}-\Delta\right)}\right)^{2}-1\right] .
$$

Using the definition of $v$, Eq. (A68), we finally obtain

$$
\phi_{0}^{\prime \prime}\left(a^{*}\right)=\frac{\tau_{a}^{2} \mu \Delta\left[2 a^{*}\left(\mu-a^{*}+\Delta\right)-\mu \Delta\right]}{2 \hat{D} a^{* 2} T^{*}\left(a^{*}-\Delta\right)^{2}} .
$$

\section{Normalization condition}

It remains to determine the prefactor $\psi_{0}\left(a^{*}\right)$. This quantity can be obtained from the known deterministic firing rate,

$$
-\lim _{\epsilon \rightarrow 0} \epsilon \hat{D} \int_{0}^{\infty} \mathrm{d} a \partial_{V} P\left(V_{\mathrm{th}}, a\right)=\frac{1}{T^{*}}
$$

[cf. Eq. (A6)]. It turns out that a finite limit can be achieved if the normalization factor $\mathcal{N}$ in Eq. (A14) scales like $1 / \sqrt{\epsilon}$, which is in line with our choice in Eq. (A15).

Using Eq. (A61) yields

$$
\begin{aligned}
\frac{1}{T^{*}} & =-\epsilon \hat{D} \int_{0}^{\infty} \mathrm{d} a \frac{N(a)}{\epsilon^{3 / 2}} \\
& =\frac{1}{\sqrt{2 \pi \epsilon}} \int_{0}^{\infty} \mathrm{d} a(\mu-a) \Psi\left(V_{\mathrm{th}}, a\right) \exp \left(-\frac{\Phi\left(V_{\mathrm{th}}, a\right)}{\epsilon}\right) .
\end{aligned}
$$

For small $\epsilon \ll 1$, the integrand is strongly peaked at $a=a^{*}-$ $\Delta$. Using Laplace's method (see, e.g., [40]), the prefactor of the exponential can be taken at $a=a^{*}-\Delta$ and can be pulled in front of the integral. Furthermore, $\Phi\left(V_{\mathrm{th}}, a\right)$ is expanded to 
lowest order in $\delta a=a-\left(a^{*}-\Delta\right)$ :

$$
\Phi\left(V_{\mathrm{th}}, a\right)=\frac{1}{2} \Phi_{a a}\left(V_{\mathrm{th}}, a^{*}-\Delta\right)\left(a-a^{*}+\Delta\right)^{2} .
$$

Finally, the lower integration bound is extended to $-\infty$ because negative values $a$ do not contribute to the integral for small $\epsilon$. Thus, Eq. (A73) takes the form

$$
\begin{aligned}
\frac{1}{T^{*}}= & \frac{1}{\sqrt{2 \pi \epsilon}}\left(\mu-a^{*}+\Delta\right) \Psi\left(V_{\mathrm{th}}, a^{*}-\Delta\right) \\
& \times \int_{-\infty}^{\infty} \mathrm{d} a \exp \left(-\frac{\Phi_{a a}\left(V_{\mathrm{th}}, a^{*}-\Delta\right)}{2 \epsilon}\left(a-a^{*}+\Delta\right)^{2}\right) \\
= & \frac{\mu-a^{*}+\Delta}{\sqrt{\Phi_{a a}\left(V_{\mathrm{th}}, a^{*}-\Delta\right)}} \psi\left(T^{*}, a^{*}\right) .
\end{aligned}
$$

Thus, due to our scaling of $\mathcal{N}(\epsilon), \epsilon$ has canceled out, leading to a finite deterministic limit of the firing rate.

The second derivative of $\Phi$ with respect to $a$ in Eq. (A74) is taken on the limit cycle and on the threshold line, which corresponds to $s=a^{*}$ and $\tau=T^{*}$. For general $\tau, \Phi_{a a}$ is given on the limit cycle by

$$
\begin{aligned}
\left.\Phi_{a a}\right|_{\mathcal{C}} ^{*} & =\partial_{a} q\left(\tau, a^{*}\right)=\frac{\partial q\left(\tau, a^{*}\right)}{\partial \tau}\left(\frac{\partial \tau}{\partial a}\right)_{V} \\
& +\left.\frac{\partial q\left(\tau, a^{*}\right)}{\partial s}\left(\frac{\partial s}{\partial a}\right)_{V}\right|_{V=V\left(\tau, s=a^{*}\right)} .
\end{aligned}
$$

From Eqs. (A39) and (A46) it follows that $\partial q\left(\tau, a^{*}\right) / \partial \tau=0$ because $p_{0}\left(a^{*}\right)=0$ and $\phi_{0}\left(a^{*}\right)=0$; hence the first term in Eq. (A75) vanishes. For the second term it holds that

$$
\frac{\partial q\left(\tau, a^{*}\right)}{\partial s}=\phi_{0}^{\prime \prime}\left(a^{*}\right)\left[e^{\tau / \tau_{a}}-\frac{a^{*}}{\mu-a^{*}}\left(1-e^{\tau / \tau_{a}}\right)\right]
$$

where Eqs. (A46) and (A69) have been used. In order to compute $(\partial s / \partial a)_{V}$ we regard $V$ as a function of the variables $s$ and $a$, which is given by Eq. (A53). The partial derivatives of this function at $s=a^{*}$ are

$$
\begin{aligned}
\left(\frac{\partial V}{\partial a}\right)_{s=a^{*}} & =\tau_{a}\left(1-\frac{\mu}{a}\right), \\
\left.\left(\frac{\partial V}{\partial s}\right)_{a}\right|_{s=a^{*}} & =\tau_{a}\left(\frac{\mu}{a^{*}}+2 \hat{D} p_{0}^{\prime}\left(a^{*}\right) \ln \frac{a^{*}}{a}-1\right) .
\end{aligned}
$$

Using Eqs. (A69) and (A68) and substituting $a=a^{*} e^{-\tau / \tau_{a}}$ results in

$$
\begin{aligned}
\left(\frac{\partial s}{\partial a}\right)_{V=V\left(\tau, s=a^{*}\right)} & =-\left.\frac{\left(\frac{\partial V}{\partial a}\right)_{s}}{\left(\frac{\partial V}{\partial s}\right)_{a}}\right|_{s=a^{*}, a=a^{*} e^{-\frac{\tau}{\tau a}}} \\
& =\frac{\mu e^{\tau / \tau_{a}}-a^{*}}{\left(\mu-a^{*}\right)(\nu \tau+1)} .
\end{aligned}
$$

Thus, it follows that

$$
\begin{aligned}
\left.\Phi_{a a}\right|_{\mathcal{C}^{*}}= & \frac{\mu e^{\tau / \tau_{a}}-a^{*}}{\left(\mu-a^{*}\right)(\nu \tau+1)} \\
& \times\left[e^{\tau / \tau_{a}}-\frac{a^{*}}{\mu-a^{*}}\left(1-e^{\tau / \tau_{a}}\right)\right] \phi_{0}^{\prime \prime}\left(a^{*}\right) .
\end{aligned}
$$

It can be shown that the prefactor in front of $\phi_{0}^{\prime \prime}\left(a^{*}\right)$ becomes unity for $\tau=T^{*}$. In other words, we have found that $\Phi$ exhibits the same shape at the reset and the threshold:

$$
\Phi_{a a}\left(V_{\mathrm{th}}, a^{*}-\Delta\right)=\phi_{0}^{\prime \prime}\left(a^{*}\right) .
$$

Substituting the last equation into Eq. (A74) and using Eqs. (A67) and (A70) yields

$$
\psi_{0}\left(a^{*}\right)=\frac{\sqrt{\phi_{0}^{\prime \prime}\left(a^{*}\right)}}{\left|\mu-a^{*}\right| T^{*}} .
$$

[1] C. W. Gardiner, Handbook of Stochastic Methods (SpringerVerlag, Berlin, 1985).

[2] F. Jülicher, K. Dierkes, B. Lindner, J. Prost, and P. Martin, Eur. Phys. J. E 29, 449 (2009).

[3] J. Grasman and J. B. T. M. Roerdink, J. Stat. Phys. 54, 949 (1989).

[4] B. Lindner and L. Schimansky-Geier, Phys. Rev. E 60, 7270 (1999).

[5] E. M. Izhikevich, Dynamical Systems in Neuroscience: The Geometry of Excitability and Bursting (MIT Press, Cambridge, MA, 2007).

[6] W. Gerstner, W. M. Kistler, R. Naud, and L. Paninski, Neuronal Dynamics: From Single Neurons to Networks and Models of Cognition (Cambridge University Press, Cambridge, UK, 2014).

[7] A. N. Burkitt, Biol. Cybern. 95, 1 (2006).

[8] Y. V. Ushakov, A. A. Dubkov, and B. Spagnolo, Phys. Rev. E 81, 041911 (2010).

[9] L. Badel, S. Lefort, R. Brette, C. C. Petersen, W. Gerstner, and M. J. Richardson, J. Neurophysiol. 99, 656 (2008).

[10] W. Gerstner and R. Naud, Science 326, 379 (2009).

[11] N. Brunel and S. Sergi, J. Theor. Biol. 195, 87 (1998).
[12] T. A. Engel, B. Helbig, D. F. Russell, L. Schimansky-Geier, and A. B. Neiman, Phys. Rev. E 80, 021919 (2009).

[13] T. Schwalger, F. Droste, and B. Lindner, J. Comput. Neurosci. 39, 29 (2015).

[14] S. Ostojic, G. Szapiro, E. Schwartz, B. Barbour, N. Brunel, and V. Hakim, J. Neurosci. 35, 7056 (2015).

[15] N. Brunel, V. Hakim, and M. J. E. Richardson, Phys. Rev. E 67, 051916 (2003).

[16] J. Benda and A. V. M. Herz, Neural Comput. 15, 2523 (2003).

[17] Y.-H. Liu and X.-J. Wang, J. Comput. Neurosci. 10, 25 (2001).

[18] S. A. Prescott and T. J. Sejnowski, J. Neurosci. 28, 13649 (2008).

[19] J. Benda, L. Maler, and A. Longtin, J. Neurophysiol. 104, 2806 (2010).

[20] T. Schwalger and B. Lindner, Front. Comput. Neurosci. 7, 164 (2013).

[21] P. E. Latham, B. J. Richmond, P. G. Nelson, and S. Nirenberg, J. Neurophysiol. 83, 808 (2000).

[22] G. La Camera, A. Rauch, H. Lüscher, W. Senn, and S. Fusi, Neural Comput. 16, 2101 (2004).

[23] M. J. E. Richardson, Phys. Rev. E 80, 021928 (2009). 
[24] M. Augustin, J. Ladenbauer, and K. Obermayer, Front. Comput. Neurosci. 7, 9 (2013).

[25] G. K. Ocker and B. Doiron, J. Neurophysiol. 112, 340 (2014).

[26] L. Hertäg, D. Durstewitz, and N. Brunel, Front. Comput. Neurosci. 8, 116 (2014).

[27] T. Schwalger, K. Fisch, J. Benda, and B. Lindner, PLoS Comput. Biol. 6, e1001026 (2010).

[28] M. Steriade, I. Timofeev, and F. Grenier, J. Neurophysiol. 85, 1969 (2001).

[29] A. Destexhe, M. Rudolph, and D. Paré, Nat. Rev. Neurosci. 4, 739 (2003).

[30] M. R. DeWeese and A. M. Zador, J. Neurosci. 26, 12206 (2006).

[31] R. Brette, Z. Piwkowska, C. Monier, M. Rudolph-Lilith, J. Fournier, M. Levy, Y. Frégnac, T. Bal, and A. Destexhe, Neuron 59, 379 (2008).

[32] M. Okun, A. Naim, and I. Lampl, J. Neurosci. 30, 4440 (2010).

[33] T. Hromádka, A. M. Zador, and M. R. DeWeese, J. Neurophysiol. 109, 1989 (2013).

[34] A. Y. Y. Tan, Y. Chen, B. Scholl, E. Seidemann, and N. J. Priebe, Nature 509, 226 (2014).

[35] K. Fisch, T. Schwalger, B. Lindner, A. Herz, and J. Benda, J. Neurosci. 32, 17332 (2012).

[36] C. Bauermeister, T. Schwalger, D. Russell, A. Neiman, and B. Lindner, PLoS Comp. Biol. 9, e1003170 (2013).

[37] T. Schwalger, D. Miklody, and B. Lindner, Eur. Phys. J. Spec. Topics 222, 2655 (2013).

[38] E. Urdapilleta, Phys. Rev. E 84, 041904 (2011).
[39] T. Schwalger, Ph.D. thesis, Humboldt-Universität zu Berlin (2013); http://edoc.hu-berlin.de/docviews/ abstract.php?id $=40328$.

[40] C. M. Bender and S. A. Orszag, Advanced Mathematical Methods for Scientists and Engineers. I: Asymptotic Methods and Perturbation Theory, Vol. 1, 1st ed. (Springer, Berlin, 1999).

[41] Z. Schuss, Theory and Applications of Stochastic Processes: An Analytical Approach (Springer, Berlin, 2009).

[42] R. Graham and T. Tél, Phys. Rev. A 33, 1322 (1986).

[43] L. F. Abbott and C. van Vreeswijk, Phys. Rev. E 48, 1483 (1993).

[44] E. Weisstein, From MathWorld-A Wolfram Web Resource (2012); http://mathworld.wolfram.com/LambertW-Function. html.

[45] G. G. Izús, R. R. Deza, and H. S. Wio, Phys. Rev. E 58, 93 (1998).

[46] D. Valenti, G. Augello, and B. Spagnolo, Eur. Phys. J. B 65, 443 (2008).

[47] H. P. McKean, Adv. Math. 4, 209 (1970).

[48] L. F. Abbott and T. B. Kepler, in Statistical Mechanics of Neural Networks, edited by L. Garrido (Springer-Verlag, Berlin, 1990), pp. 5-18.

[49] B. Lindner and L. Schimansky-Geier, Phys. Rev. E 61, 6103 (2000).

[50] B. Lindner, Coherence and Stochastic Resonance in Nonlinear Dynamical Systems (Logos-Verlag, Berlin, 2002).

[51] N. Fourcaud and N. Brunel, Neural Comput. 14, 2057 (2002). 